\title{
A New Robust Controller with Applications to Bioreactors
}

\author{
Alejandro Rincón, ${ }^{1}$ Christian Camilo Erazo Ordoñez, ${ }^{2}$ Felipe Londoño, ${ }^{2}$ \\ Gerard Olivar Tost, ${ }^{2}$ and Fabiola Angulo ${ }^{2}$ \\ ${ }^{1}$ Facultad de Ingeniería y Arquitectura, Universidad Católica de Manizales, Carrera 23, No. 60-63, Manizales 170002, Colombia \\ ${ }^{2}$ Universidad Nacional de Colombia, Sede Manizales, Facultad de Ingeniería y Arquitectura, Departamento de Ingeniería Eléctrica, \\ Electrónica y Computación, Percepción y Control Inteligente, Bloque Q, Campus La Nubia, Manizales 170003, Colombia
}

Correspondence should be addressed to Fabiola Angulo; fangulog@unal.edu.co

Received 27 December 2013; Accepted 10 April 2014; Published 11 May 2014

Academic Editor: ShengJun Wen

\begin{abstract}
Copyright (C) 2014 Alejandro Rincón et al. This is an open access article distributed under the Creative Commons Attribution License, which permits unrestricted use, distribution, and reproduction in any medium, provided the original work is properly cited.

In this work an anaerobic digester is controlled using input-output linearization and Lyapunov-like function methods. It is assumed that model parameters are unknown, time-varying, and bounded, and upper or lower bounds are also unknown. To tackle the effect of input saturation, a state observer is designed. The tracking and observer errors are defined in terms of the noisy measured output instead of ideal output, given by the mathematical model. The design of the observer mechanism and the update laws is based on the Lyapunov-like function technique, whereas the design of the control law is based on the input-output linearization method. In this paper two important properties of the controlled system are proven. First, the observer error converges asymptotically to a residual set whose size is user-defined, and such convergence is not disrupted, neither by the input saturation nor by the parameter uncertainties. Second, when the control input is nonsaturated the tracking error converges to a residual set whose size is user-defined. The model parameter uncertainties are included to prove the convergence of errors. Finally, a numerical example to validate the developed control is presented.
\end{abstract}

\section{Introduction}

Nonlinear control techniques have been widely developed in the last years. The adaptive control is perhaps one of the most important techniques to control systems, mainly due to its ability to compensate for the parametric model uncertainties. In [1-5], an adaptive fuzzy tracking control is designed to control a class of stochastic nonlinear systems. In $[1,2]$ it is proven that the closed loop signals are bounded in probability, and the tracking error eventually converges to a residual set whose size is not known a priori or predefined by the user. In [3-5], a fuzzy state observer is included, and the convergence of the output and the tracking error are guarantee to residual sets whose sizes cannot be predefined by the user.

High gain observer is effective in estimating system states and output derivatives and in rejecting modelling disturbances in absence of noise [6-9]. Nevertheless, state estimation is degraded in the presence of measurement noise and gets worse for large observer gain $[8,9]$. In $[7,8]$, nonlinear plant models in state-space form and in controllable form are considered, respectively. Both plant models involve known constant coefficients. The real output is defined as the first state and is measured, whereas the other states are not. The output measurement is expressed as the sum of the real output plus a bounded of the noisy parameter measurement. The observer depends on the difference between the noisy output measurement and the output estimate. The stability analysis indicates that the state estimation error converges to a residual set whose size is not known a priori; even more, the coefficients of the plant model are required to be known.

Interval observers provide an upper and a lower bound (intervals) for each unmeasured state variable. Upper and lower bounds of some plant parameters are introduced in the observer mechanism, leading to two observer equations and two estimated states for each unmeasured state. Such 
estimated states constitute intervals for each unmeasured state and can be used to develop a robust controller [10-12].

Nevertheless, interval observers have the following features: (i) several upper and lower bounds of the plant model parameters are required to be known; (ii) overestimated values of the unmeasured states can result if uncertainty on the feed concentrations is broad; and (iii) upper or lower bounds of the noise model parameters are required to be known in the case of some schemes as that in [11].

In the robust controller presented in [13], a plant in parametric pure feedback form is considered. It is assumed that each measurement of the plant states is corrupted by noise and is expressed as the real value of the state variable with additive and multiplicative noise parameters. The noise parameters are unknown, time-varying, bounded, and their time derivatives are unknown and bounded. Therefore, if such noise model is differentiated, the time derivative of the state measurement is the time derivative of the real state variable with additive and multiplicative parameters. The states resulting from the backstepping state transformation are defined in terms of the noisy measurements instead of the real states. Hence, the differentiation of each quadratic function involves the differentiation of each noise model. Thus, the control law formulation is based on the noisy measurement instead of the real states. As the stability analysis indicates, the measured and real states remain bounded but do not converge to the expected residual set. Therefore, the convergence of the tracking error to a residual set of user-defined size is not achieved. The approach of [13] for considering the noise model will be used in this work.

In the present paper it is assumed that model parameters are unknown, time-varying, and bounded, and upper or lower bounds are also unknown. Constant upper bounds are established for the biological concentrations and reaction rate terms and are valid for the case of nonnegative dilution rate and time-varying but bounded model parameters. These bounds are used in the control design and in proving the boundedness and convergence of the closed loop signals. The tracking and observer errors are defined in terms of the noisy measured output instead of the real output. In fact, the observer error is defined as the difference between the measured and the estimated output. A state observer allows us to tackle the effect of input saturation. The design of the observer mechanism and the update laws is based on the Lyapunovlike function technique, whereas the design of the control law is based on the input-output linearization method. The main difference between Lyapunov functions and Lyapunov-like functions is the condition to be zero. The Lyapunov functions must be zero only in the origin of the state space and greater than zero outside the origin; the Lyapunov-like functions must be zero in the origin of the state space as well as in a predefined region, and it must be positive outside of this region. Other conditions such as continuity, differentiability, and being semidefinite derivative must be satisfied by the Lyapunov-like functions. The following benefits are achieved with the proposed controller:

Bf 1.1 the exact values of the plant and noise model parameters are not required to be known, and although a nominal value of the influent concentration is required to be known, other upper or lower bounds of model parameters are not required to be known;

Bf 1.2 the parameter uncertainty is taken into account in the stability analysis, such that the convergence and boundedness properties are not disrupted by such uncertainty;

Bf 1.3 the observer error converges asymptotically to a residual set whose size is user-defined, and such convergence is not disrupted, neither by the input saturation nor the parameter uncertainty;

Bf 1.4 discontinuous signals are avoided in the control and update laws;

Bf 1.5 the updated parameters are bounded, so that parameter drifting is absent, despite input saturation;

Bf 1.6 when the control input is not saturated, it is guaranteed that the tracking error converges to a residual set whose size is user-defined.

The major contributions with respect to closely related works are the benefits $\mathrm{Bf} 1.1, \mathrm{Bf} 1.2$, and $\mathrm{Bf} 1.3$, which are significant contributions with respect to the aforementioned control schemes that tackle the effect of measurement noise, for instance $[7,8,11,13]$.

This paper is organized as follows. Section 2 shows characteristics, assumptions, and model of the plant, the goal of the control design, and the bounded nature of the model concentrations and reaction rate terms. Section 3 presents the control design, including the formulation of the control law, the update law, and the state observer. Section 4 presents the proof for (i) the bounded nature of the closed loop signals despite input saturation, (ii) the convergence of the observer error to a residual set of user-defined size despite input saturation, and (iii) the convergence of the tracking error to a residual set of user-defined size, when the control input is not saturated. Section 5 shows a simulation example and finally Section 6 shows the discussion and conclusions.

\section{The Plant Model and Control Goal}

2.1. Plant Model. The upflow anaerobic fixed bed reactor of [14] is considered, whose mass-balance model is

$$
\begin{aligned}
\dot{X}_{1} & =X_{1}\left(\mu_{1}-\alpha D\right), \\
\dot{X}_{2} & =X_{2}\left(\mu_{2}-\alpha D\right), \\
\dot{S}_{1} & =\left(S_{1}^{\text {in }}-S_{1}\right) D-k_{1} \mu_{1} X_{1}, \\
\dot{S}_{2} & =\left(S_{2}^{\text {in }}-S_{2}\right) D+k_{2} \mu_{1} X_{1}-k_{3} \mu_{2} X_{2}, \\
\mu_{1}\left(S_{1}\right) & =\mu_{1 \max } \frac{S_{1}}{S_{1}+K_{S_{1}}}, \\
\mu_{2}\left(S_{2}\right) & =\mu_{2 \max } \frac{S_{2}}{S_{2}+K_{s_{2}}+\left(1 / K_{I_{2}}\right) S_{2}^{2}},
\end{aligned}
$$

where $X_{1}, S_{1}, X_{2}$, and $S_{2}$ represent the concentrations of acidogenic biomass, methanogenic biomass, chemical oxygen 
demand, and volatile fatty acids (VFA); $D$ is the dilution rate; $\alpha$ is the proportion of biomass not attached to the reactor; $\mu_{1}$ and $\mu_{2}$ are the specific growth rates; $k_{1}, k_{2}$, and $k_{3}$ are yield coefficients; $\mu_{1 \text { max }}, K_{S_{1}}, \mu_{2 \text { max }}, K_{S_{2}}$, and $K_{I_{2}}$ are kinetic parameters. As in [15], the concentration $S_{2}$ and the dilution rate $D$ are chosen as the output to be controlled and the control input. The characteristics of model parameters, state variables, influent concentrations, and dilution rate have been depicted in literature on anaerobic digestion and bioreactors and are considered in this work.

Characteristic 1. The value of $D$ is available online $[12,16]$, whereas $S_{1}, X_{1}$, and $X_{2}$ are unknown. This is in agreement with $[12,15-17]$.

Characteristic 2. The concentrations $X_{1}, X_{2}, S_{1}$, and $S_{2}$ are nonnegative $[16,18,19]$.

Characteristic 3. The inflow substrate concentration $S_{1}^{\text {in }}$, the yield coefficients, the proportion $\alpha$, and the kinetic parameters are unknown, varying, and bounded, and upper bounds of their values are unknown $[12,20,21]$. In this work we consider the following notation: $0<k_{1 \min } \leq k_{1}, k_{2} \leq k_{2 \max }$, $0<k_{3 \min } \leq k_{3} \leq k_{3 \max }, 0<k_{6 \min } \leq k_{6} \leq k_{6 \max }$, $S_{1}^{\text {in }} \leq S_{1 i \text { max }}, S_{2}^{\text {in }} \leq S_{2 i \max }, \alpha \geq \alpha_{\min }, \mu_{2 \max } \leq \bar{\mu}_{2 \max }, K_{S_{2}} \geq$ $K_{S_{2} \text { min }}, \leq K_{I_{2} \text { min }} \leq K_{I_{2} \text { max }}$, where $k_{1 \text { min }}, k_{2 \text { max }}, k_{3 \text { min }}, k_{3 \text { max }}$, $k_{6 \text { min }}, S_{1 i \text { max }}, S_{2 i \text { max }}, k_{6 \text { max }}, \alpha_{\text {min }}, \bar{\mu}_{2 \text { max }}, K_{S_{2} \min }$, and $K_{I_{2} \min }$ are unknown positive constants, whereas $k_{1}, k_{2}, k_{3}, k_{6}, S_{1}^{\text {in }}$, $\alpha, \mu_{2 \max }, K_{S_{2}}$, and $K_{I_{2}}$ are unknown, varying and bounded.

Characteristic 4. The dilution rate $D$ is constrained between known bounds $u_{\min }$ and $u_{\max }: 0 \leq u_{\min } \leq D \leq u_{\max } \forall t \geq t_{o}$, where $u_{\text {min }}$ is a nonnegative constant, and $u_{\max }$ is a positive constant $[15,22]$.

Characteristic 5. The values of the specific growth rates $\mu_{1}$ and $\mu_{2}$ are unknown to the controller $[15,22]$.

Characteristic 6. The inflow substrate concentration $S_{2}^{\text {in }}$ is unknown, varying, and bounded $[15,16]$, but a nominal value is known, defined as $\bar{S}_{2}^{\text {in }}$, such that $\bar{S}_{2}-S_{2 m}>0 \forall t>t_{0}$. This is in agreement with $[14,15,18]$.

The following assumption is made.

Assumption 1. The measurement of $S_{2}$ is corrupted by noise with multiplicative and additive noise. This is in agreement with $[10,16,23]$. As in [13] the noise model is given by $S_{2 m}=$ $a_{1} S_{2}+a_{2}$, where $S_{2 m}$ is the known measurement of $S_{2}$ and $S_{2}$ is unknown; $a_{1}$ and $a_{2}$ are unknown varying parameters bounded by unknown constants, and their time derivatives are bounded by unknown constants too; and $a_{1}$ is positive and bounded away from zero.

Remark 2. The fact that the inflow substrate concentration $S_{2}^{\text {in }}$ is unknown is made in order to allow reducing costs. The online measurement of $S_{2}^{\text {in }}$ would require an online and accurate sensor. In contrast, the offline measurement of $S_{2}^{\text {in }}$ implies lower costs and allows us to define the upper bound $\bar{S}_{2}^{\text {in }}$.
For the sake of notational simplicity, the following changes of variables are made: the output $S_{2}$ is noted as $y$; the input control $D$ is noted as $u$; the measured output signal $S_{2 m}$, which is corrupted by noise, is noted as $y_{m}$. At this point, it is worth to note that through the paper the real output $y$ is different from the measured output $y_{m}$ which is the value used in the design of the observer and the controller. With the aim to rewrite (4) the following changes are made: $S_{2}^{\text {in }}-y$ is noted as $b$ and represents the control gain and $k_{2} \mu_{1} X_{1}-$ $k_{3} \mu_{2} X_{2}$ is noted as $a_{0}$. With this notation the dynamics of VFA concentration in (4) is rewritten in (6) and the dynamics of measured VFA is expressed in (7):

$$
\begin{gathered}
\dot{y}=b u+a_{o} \\
y_{m}=a_{1} y+a_{2},
\end{gathered}
$$

where

$$
b=S_{2}^{\text {in }}-y, \quad a_{o}=k_{2} \mu_{1} X_{1}-k_{3} \mu_{2} X_{2} .
$$

Characteristics 1 to 6 and Assumption 1 lead to some properties for $a_{o}, a_{1}, a_{2}, b, S_{2}^{\text {in }}$, and $u$ :

(Pi) the control input $u$ satisfies $u_{\text {min }} \leq u \leq u_{\max }$;

(Pii) since $S_{2}$ and $S_{2}^{\text {in }}$ are unknown, $b$ is unknown;

(Piii) since $X_{1}$ and $X_{2}$ are unknown and varying, then $a_{o}$ is unknown and varying;

(Piv) $S_{2}^{\text {in }}$ is upper bounded by a known positive constant that satisfies $\bar{S}_{2}^{\text {in }}-y_{m}>0 \forall t \geq t_{o}$;

(Pv) The parameters $1 / a_{1}, a_{1}, a_{2}, \dot{a}_{1}$, and $\dot{a}_{2}$ are upper bounded by unknown positive constants.

Remark 3. The property $(\mathrm{Pv})$ means the following: (i) the parameter $a_{1}$ is positive and bounded away from zero and (ii) the varying parameters $a_{1}$ and $a_{2}$ do not exhibit abrupt step type changes.

\subsection{Control Goal. Let}

$$
\begin{aligned}
e(t) & =y_{m}(t)-y_{d}(t), \\
\dot{y}_{d}(t) & =-a_{m} y_{d}(t)+a_{m} r(t), \\
\Omega_{e} & =\left\{e \in \mathbb{R}:|e| \leq C_{b e}\right\},
\end{aligned}
$$

where $e(t):=e$ is the tracking error, $y_{d}(t):=y_{d}$ is the desired output, $r(t):=r$ is the command signal, which is user-defined, $\Omega_{e}$ is a residual set, and $a_{m}$ and $C_{b e}$ are positive constants defined by the user. The goal of the control design is to formulate a control law, an update law, and an observer mechanism for the plant model (1) to (4), subject to Characteristics 1 to 6 and Assumption 1, such that (CGi) the observer error converges to a residual predefined size set despite the input saturation, (CGii) the tracking error $e(t)$ converges asymptotically to the residual set $\Omega_{e}$ when the control input is not saturated, (CGiii) the controller does not involve discontinuous signals, (CGiv) the control law, the updating mechanism, and the observer mechanism provide bounded values of the control input, updated parameter vector, and output estimate. 
2.3. Boundedness of the Anaerobic Digester States under NonNegative Dilution Rate. The bounded nature of the digester states and reaction rate terms are commonly used in control design and stability analysis as can be noticed in [16, 24-26]. In the present work, upper constant bounds are established for the digester states and reaction rate terms, in order to allow the controller design and the stability analysis.

Lemma 4. Consider the plants (1) to (4), subject to Characteristics 1 to 6 and Assumption 1. The plant model concentrations and reaction rate terms exhibit the following characteristics: (i) the concentration of acidogenic biomass and COD, that is, $X_{1}$ and $S_{1}$, are upper bounded by positive constants that are unknown to the controller, (ii) the reaction rate term $k_{2} \mu_{1} X_{1}$ is upper bounded as $k_{2} \mu_{1} X_{1} \leq \bar{\kappa}_{\mu_{1} X_{1}}$, where $\bar{\kappa}_{\mu_{1} X_{1}}$ is a positive constant that is unknown to the controller, (iii) the concentrations of methanogenic biomass and VFA are bounded as follows: $X_{2} \leq \bar{\kappa}_{X_{2}}, S_{2} \leq \bar{\kappa}_{S_{2}}$, where $\bar{\kappa}_{X_{2}}, \bar{\kappa}_{S_{2}}$ are positive constants that are unknown to the controller, and (iv) the term $\mu_{2} X_{2}$ is bounded as follows: $\mu_{2} X_{2} \leq \bar{\kappa}_{\mu_{2} X_{2}}$, where $\bar{\kappa}_{\mu_{2} X_{2}}$ is a positive constant that is unknown to the controller.

The proof is presented in Appendix A. In the control design and stability analysis, the above lemma will be used to tackle the lack of knowledge on the term $a_{o}=k_{2} \mu_{1} X_{1}-$ $k_{3} \mu_{2} X_{2}$, to prove the bounded nature of the closed loop signals and to prove the asymptotic convergence of the observer error.

\section{Control Design}

In this section, the state observer, the control law, and the update law are formulated considering the plants (1) to (4), subject to Characteristics 1 to 6 , Assumption 1, and goals (CGi) to (CGiv). Discontinuous signals are avoided in the control design, because such signals lead to discontinuous vectorial field and undesired effects in the closed loop system, that is, (i) trajectory unicity may be lost [27], (ii) the state trajectories may undergo sliding motion and consequently state chattering along the discontinuity surface $[27,28]$, and (iii) input chattering may occur [22]. Input chattering consists of a commutation component in the control input with large commutation rate [22]. It may result in high power consumption and wear of mechanical components $[29,30]$.

As mentioned in $[22,31]$, the input saturation may lead to "integrator windup" phenomenon. In this case, the integral term may exhibit an excessive increase when the input gets saturated, what leads to slow convergence, overshoot, and large steady state of the tracking error [22]. This can be remedied by means of state observers. In the controllers presented in $[16,22,32]$ the observer error convergence is not affected by input saturation. In [32], the convergence of the tracking error is guaranteed when the input does not get saturated but is not guaranteed on the contrary case.

In view of the above discussion, a state observer is considered in the present work and discontinuous signals are avoided in the controller mechanism. The state observer allows us to handle the effect of control input saturation and avoid excessive increases of updating parameters when the input gets saturated. The lack of knowledge of the unknown varying but bounded parameters of the plant and noise models, and constant upper bounds of the plant and noise model parameters, is tackled by means of an updated parameter vector, which is provided by an update law. The Lyapunov-like function method is used instead of the classical direct Lyapunov method, in order to design a controller that does not involve discontinuous signals and leads to adequate boundedness and convergence properties (see $[28,32-34])$. When a state observer is used, the state dependent quadratic form is defined in terms of the observer error instead of the tracking error (see [35]). A truncation is introduced in the definition of the quadratic form, in order to avoid discontinuous signals in the observer mechanism, update law, and control law and avoid the aforementioned state and input chattering phenomena. The time derivative of the quadratic form is rewritten in terms of unknown constant upper bounds, and such bounds are expressed in terms of updated parameters, in order to handle the uncertainty on plant model parameters, noise model parameters, and upper bounds. The Lyapunov-like function depends on the closed loop states, that is, the observer error and the parameter updating error. The formulation of the observer and the updating law expressions is such that the time derivative of the Lyapunov-like function is upper-bounded by a function that exhibits certain properties, which in turn lead to the required boundedness of the closed loop signals and convergence of the observer error. The formulation of the control law is such that the estimated output converges asymptotically to the desired output, for the time lapses during which the control input does not get saturated. As a result of the control design and stability analysis, the tracking error $e$ converges asymptotically to the residual set $\Omega_{e}$ for such time lapses. The constant upper bounds established in Section 2.3 are used in the control design and in proving the boundedness and convergence properties of the closed loop signals. As a result, the following facts are guaranteed: (i) the observer error converges asymptotically to a residual set whose size is user-defined, (ii) the updated parameters are bounded, so that parameter drifting is avoided, (iii) the control law, the output estimate, and the closed loop signals are bounded in closed loop, and (iv) the tracking error converges towards a residual set whose size is user-defined, when the input is not saturated. In summary, the benefits Bf 1.1 to Bf 1.6 are achieved.

The steps for the formulation of the observer, the update law, and the control law are summarized as follows: (i) define the observer error and differentiate it with respect to time; (ii) formulate a quadratic-like function that depends on the observer error, and differentiate such function with respect to time; (iii) express the unknown varying coefficients of the plant model and measurement model in terms of an updated parameter vector and an updating error vector; (iv) define the observer, such that the time derivative of the quadratic function involves terms that contribute to the required stability properties; (v) formulate the Lyapunovlike function and differentiate it with respect to time; (vi) formulate the updating law; and (vii) formulate the control law. The above steps are developed as the following. 
3.1. Step 1. In this step, the observer error is defined and differentiated with respect to time. Let

$$
z=y_{m}-\widehat{y}
$$

where $y_{m}$ is the measured output and fulfills (7), whereas $\widehat{y}$ is the estimated output, which is provided by an state observer that will be defined later. Differentiating, with respect to time, yields

$$
\dot{z}=\dot{y}_{m}-\dot{\hat{y}}
$$

Differentiating (7), with respect to time, and using (6) yield

$$
\begin{aligned}
\dot{y}_{m} & =a_{1} \dot{y}+\dot{a}_{1} y+\dot{a}_{2} \\
& =a_{o} a_{1}+\dot{a}_{1} y+\dot{a}_{2}+a_{1} b u .
\end{aligned}
$$

Substituting (14) into (13) yields

$$
\dot{z}=\dot{a}_{1} y+a_{o} a_{1}+\dot{a}_{2}-\dot{\hat{y}}+a_{1} b u .
$$

and since $y$ is unknown, it should be expressed in terms of $y_{m}$. Solving (7) for $y$ yields

$$
y=\frac{1}{a_{1}} y_{m}-\frac{a_{2}}{a_{1}} .
$$

Substituting in (15) yields

$$
\dot{z}=-\frac{a_{2}}{a_{1}} \dot{a}_{1}+a_{o} a_{1}+\dot{a}_{2}+\frac{\dot{a}_{1}}{a_{1}} y_{m}+a_{1} b u-\dot{\hat{y}} .
$$

3.2. Step 2. In this step, a quadratic function that depends on the observer error is formulated and differentiated with respect to time. The truncated function, appearing in $[28,32-$ 34], allows us to obtain (i) adequate time derivative of the Lyapunov-like function, (ii) adequate stability properties, and (iii) continuous controller. The Lyapunov-like function is defined as in $[28,32-34]$ :

$$
V_{z}(z)= \begin{cases}\left(\frac{1}{2}\right)\left(z-C_{b z}\right)^{2} & \text { if } z \geq C_{b z} \\ 0 & \text { if } z \in\left(-C_{b z}, C_{b z}\right) \\ \left(\frac{1}{2}\right)\left(z+C_{b z}\right)^{2} & \text { if } z \leq-C_{b z},\end{cases}
$$

where $C_{b z}$ is a positive constant whose value will be defined later, and $z$ is defined in (12). Expression (18) is based on the distance of the observer error $z$ to the boundary layer with $C_{b z}$ in width, as stated in [28]. The function $V_{z}$ (18) presents the three properties mentioned above. From (18) it follows that

$$
\begin{aligned}
& \text { (i) } V_{z} \geq 0 \\
& \text { (ii) }|z| \leq C_{b z}+\sqrt{2 V_{z}}, \\
& \text { (iii) if } V_{z} \in L_{\infty}, \quad \text { then } z \in L_{\infty} \text {, } \\
& \text { (iv) } V_{z}, \frac{\partial V_{z}}{\partial z} \text { are locally Lipschitz continuous. }
\end{aligned}
$$

The proof of (20) is shown in Appendix B. The Lipschitz continuity allows us to avoid discontinuous signals in the controller and consequently allows us to avoid loss of trajectory unicity and state and input chattering. The time derivative of the Lyapunov-like function has to be upper bounded by a function that exhibits certain properties [3234]: (TDPi) the function is not positive (TDPii), the function is zero when the observer error is inside or on the boundary of a residual set of predefined small size, and (TDPiii) the function is negative when the observer error is outside such residual set. The fulfillment of such properties implies that (i) the observer error converges asymptotically to a residual set of adequate predefined size and (ii) the control law, the updating mechanism, and the observer mechanism provide bounded signals. Differentiating (18), with respect to time, yields

$$
\begin{aligned}
& \frac{d V_{z}}{d t}=\frac{\partial V_{z}}{\partial z} \dot{z}, \\
& \frac{\partial V_{z}}{\partial z} \triangleq g_{z}= \begin{cases}z-C_{b z} & \text { if } z \geq C_{b z} \\
0 & \text { if } z \in\left(-C_{b z}, C_{b z}\right) \\
z+C_{b z} & \text { if } z \leq-C_{b z},\end{cases}
\end{aligned}
$$

where $z$ is defined in (12). Substituting (17) into (23) yields

$$
\begin{aligned}
\dot{V}_{z}= & g_{z} \dot{z}=-c_{1} g_{z}^{2} \\
& +g_{z}\left(-\frac{a_{2}}{a_{1}} \dot{a}_{1}+a_{o} a_{1}+\dot{a}_{2}+\frac{\dot{a}_{1}}{a_{1}} y_{m}+a_{1} b u-\dot{\hat{y}}+c_{1} g_{z}\right) .
\end{aligned}
$$

The notation $g_{z}$ has been introduced for notational simplicity, and the term $c_{1} g_{z}^{2}$ has been added and subtracted in order to contribute to obtain the required stability properties. Incorporating (8) and (16) in (25) yields

$$
\begin{aligned}
\dot{V}_{z}= & -c_{1} g_{z}^{2} \\
& +g_{z}\left(-\frac{a_{2}}{a_{1}} \dot{a}_{1}+a_{o} a_{1}+\dot{a}_{2}+\frac{\dot{a}_{1}}{a_{1}} y_{m}\right. \\
& \left.+\left(a_{1} S_{2}^{\text {in }}-y_{m}+a_{2}\right) u-\dot{\hat{y}}+c_{1} g_{z}\right) .
\end{aligned}
$$

3.3. Step 3. Since the lumped parameters $\left(-a_{2} a_{1} / a_{1}+a_{0} a_{1}+\dot{a}_{2}\right)$ and $\dot{a}_{1} / a_{1}, a_{1} S_{2}^{\text {in }}-\bar{S}_{2}^{\text {in }}+a_{2}$ are unknown, time-varying, and bounded, they should be expressed in terms of unknown constant upper bounds, and such bounds should be expressed in terms of an updated parameter vector and an updating error vector.

The Luenberger-type observers for anaerobic digesters may require the control gain to be known in order to cancel the control input term (see [22]). Equation (26) indicates that the cancelation of the term $\left(a_{1} S_{2}^{\text {in }}-y_{m}+a_{2}\right) u$ by the observer mechanism $\dot{\hat{y}}$ requires the term $a_{1} S_{2}^{\text {in }}-y_{m}+a_{2}$ to be known. Nevertheless, because of the uncertainty on $a_{1}$, $S_{2}^{\text {in }}$, and $a_{2}$, such cancelation is not possible. According to the experimental data shown in [14], the term $S_{2}^{\text {in }}-y_{m}=S_{2}^{\text {in }}-S_{2 m}$ 
is usually positive. Therefore, Characteristic 6 is considered, that is,

$$
\bar{b} \triangleq \bar{S}_{2}^{\text {in }}-y_{m}>0 \quad \forall t \geq t_{o},
$$

where $\bar{S}_{2}^{\text {in }}$ is a known nominal value of $S_{2}^{\text {in }}$ that fulfills the above property. Equation (26) can be rewritten as

$$
\begin{aligned}
\dot{V}_{z}= & -c_{1} g_{z}^{2} \\
& +g_{z}\left(-\frac{a_{2}}{a_{1}} \dot{a}_{1}+a_{o} a_{1}+\dot{a}_{2}+\frac{\dot{a}_{1}}{a_{1}} y_{m}\right. \\
& \left.\quad+\left(a_{1} S_{2}^{\text {in }}-y_{m}+a_{2}-\bar{b}\right) u+\bar{b} u-\dot{\hat{y}}+c_{1} g_{z}\right) .
\end{aligned}
$$

In view of (27), it follows that

$$
a_{1} S_{2}^{\text {in }}-y_{m}+a_{2}-\bar{b}=a_{1} S_{2}^{\text {in }}-\bar{S}_{2}^{\text {in }}+a_{2} .
$$

Hence, (28) can be rewritten as

$$
\begin{aligned}
\dot{V}_{z} & \\
= & -c_{1} g_{z}^{2} \\
& +g_{z}\left(-\frac{a_{2}}{a_{1}} \dot{a}_{1}+a_{o} a_{1}+\dot{a}_{2}+\frac{\dot{a}_{1}}{a_{1}} y_{m}+\left(a_{1} S_{2}^{\text {in }}-\bar{S}_{2}^{\text {in }}+a_{2}\right) u\right) \\
& +\left(\bar{b} u-\dot{\hat{y}}+c_{1} g_{z}\right) g_{z} .
\end{aligned}
$$

In accordance with [32, 33], the unknown plant model lumped parameter terms are expressed in terms of unknown positive constant upper bounds, and the uncertainty on such bounds is tackled by means of updated parameters. The term inside large parenthesis in (30) leads to

$$
\begin{aligned}
g_{z}( & \left.-\frac{a_{2}}{a_{1}} \dot{a}_{1}+a_{o} a_{1}+\dot{a}_{2}+\frac{\dot{a}_{1}}{a_{1}} y_{m}+\left(a_{1} S_{2}^{\text {in }}-\bar{S}_{2}^{\text {in }}+a_{2}\right) u\right) \\
\leq & \left|-\frac{a_{2}}{a_{1}} \dot{a}_{1}+a_{o} a_{1}+\dot{a}_{2}\right|\left|g_{z}\right|+\left|-\frac{\dot{a}_{1}}{a_{1}}\right|\left|y_{m}\right|\left|g_{z}\right| \\
& +\left|a_{1} S_{2}^{\text {in }}-\bar{S}_{2}^{\text {in }}+a_{2}\right||u|\left|g_{z}\right| .
\end{aligned}
$$

Definition (8) indicates that $a_{o}$ involves the terms $k_{2} \mu_{1} X_{1}$ and $k_{3} \mu_{2} X_{2}$. Characteristic 3 mentions that $k_{3}$ is upper bounded by a constant, Lemma A. 3 mentions that $k_{2} \mu_{1} X_{1}$ is bounded, and Lemma A.7 mentions that $\mu_{2} X_{2}$ is bounded. Therefore, the term $a_{o}$ is upper-bounded by an unknown positive constant. This statement, jointly with Characteristic 6 and property $(\mathrm{Pv})$ stated in Section 2.1, implies that the lumped parameters appearing in (31) are upper bounded by unknown positive constants:

$$
\begin{gathered}
\left|-\frac{a_{2}}{a_{1}} \dot{a}_{1}+a_{o} a_{1}+\dot{a}_{2}\right| \leq \bar{\kappa}_{1}, \quad\left|-\frac{\dot{a}_{1}}{a_{1}}\right| \leq \bar{\kappa}_{2}, \\
\left|a_{1} S_{2}^{\text {in }}-\bar{S}_{2}^{\text {in }}+a_{2}\right| \leq \bar{\kappa}_{3},
\end{gathered}
$$

where $\bar{\kappa}_{1}, \bar{\kappa}_{2}$, and $\bar{\kappa}_{3}$ are unknown positive constant bounds. Substituting the above expressions into (31), using property $(\mathrm{Pi})$ and arranging in terms of parameter and regression vectors, yields

$$
\begin{gathered}
g_{z}\left(-\frac{a_{2}}{a_{1}} \dot{a}_{1}+a_{o} a_{1}+\dot{a}_{2}+\frac{\dot{a}_{1}}{a_{1}} y_{m}+\left(a_{1} S_{2}^{\text {in }}-\bar{S}_{2}^{\text {in }}+a_{2}\right) u\right) \\
\leq \bar{\kappa}_{1}\left|g_{z}\right|+\bar{\kappa}_{2}\left|y_{m}\right|\left|g_{z}\right|+\bar{\kappa}_{3}\left|g_{z}\right| u_{\text {max }}=\varphi^{\top} \theta\left|g_{z}\right|,
\end{gathered}
$$

where

$$
\begin{aligned}
& \varphi=\left[1,\left|y_{m}\right|, u_{\max }\right]^{\top}, \\
& \theta=\left[\bar{\kappa}_{1}, \bar{\kappa}_{2}, \bar{\kappa}_{3}\right]^{\top} .
\end{aligned}
$$

Substituting (33) into (30) yields

$$
\dot{V}_{z} \leq-c_{1} g_{z}^{2}+\varphi^{\top} \theta\left|g_{z}\right|+g_{z}\left(\bar{b} u+c_{1} g_{z}-\dot{\hat{y}}\right) .
$$

The parameter vector $\theta$ can be rewritten as

$$
\theta=\widehat{\theta}-\tilde{\theta},
$$

where

$$
\widetilde{\theta} \triangleq \widehat{\theta}-\theta
$$

where $\widetilde{\theta}$ is an updating error vector and $\widehat{\theta}$ is an updated parameter vector provided by an update law that will be defined later and $\theta$ is the unknown constant vector defined in (35). Substituting (37) into (36) and arranging yields

$$
\begin{aligned}
\dot{V}_{z} \leq & -c_{1} g_{z}^{2}+g_{z}\left(\varphi^{\top} \hat{\theta} \operatorname{sgn}\left(g_{z}\right)+\bar{b} u+c_{1} g_{z}-\dot{\hat{y}}\right) \\
& -\varphi^{\top} \tilde{\theta}\left|g_{z}\right| .
\end{aligned}
$$

3.4. Step 4. The state observer $\dot{\hat{y}}$ has to be defined such that the right hand side of (39) contains the terms $-c_{1} g_{z}^{2}$ and $-\varphi^{\top} \widetilde{\theta}\left|g_{z}\right|$, and the remaining terms are canceled, such that the time derivative of the Lyapunov-like function fulfills the properties (TDPi), (TDPii), and (TDPiii) mentioned in Step 2. Expression (39) indicates that the observer mechanism $\dot{\hat{y}}$ that leads to adequate time derivative of $\dot{V}_{z}$ would contain the term $\operatorname{sgn}\left(g_{z}\right)$. The drawback is that the term $\operatorname{sgn}\left(g_{z}\right)$ is discontinuous with respect to $z$. From (24) it follows that

$$
\operatorname{sgn}\left(g_{z}\right)= \begin{cases}\operatorname{sgn}(z) & \text { if }|z|>C_{b z} \\ 0 & \text { otherwise }\end{cases}
$$

Using the following property (which is based on [34]):

$$
\begin{aligned}
& \left|g_{z}\right|=g_{z} \text { sat }_{z} \\
& \text { sat }_{z}= \begin{cases}\operatorname{sgn}(z) & \text { if }|z|>C_{b z} \\
\left(\frac{1}{C_{b z}}\right) z & \text { otherwise, }\end{cases}
\end{aligned}
$$


the discontinuity respect to $z$ is avoided. The proof of property (41) is presented in C. Introducing (41) into (39) yields

$$
\begin{aligned}
\dot{V}_{z} \leq & -c_{1} g_{z}^{2}+g_{z}\left(\varphi^{\top} \hat{\theta} \mathrm{sat}_{z}+\bar{b} u+c_{1} g_{z}-\dot{\hat{y}}\right) \\
& -\varphi^{\top} \tilde{\theta}\left|g_{z}\right| .
\end{aligned}
$$

The observer $\dot{\hat{y}}$ should cancel the effect of terms $\varphi^{\top} \hat{\theta}$ sat $_{z}, \bar{b} u$, and $c_{1} g_{z}$. Thus, $\dot{\hat{y}}$ is chosen as

$$
\dot{\hat{y}}=\varphi^{\top} \hat{\theta} \mathrm{sat}_{z}+\bar{b} u+c_{1} g_{z}
$$

Substituting into (43) yields

$$
\dot{V}_{z} \leq-c_{1} g_{z}^{2}-\varphi^{\top} \tilde{\theta}\left|g_{z}\right|
$$

3.5. Step 5. In this step, the Lyapunov-like function is formulated and differentiated with respect to time. The Lyapunovlike function is defined as $[28,32,33]$

$$
\begin{gathered}
V(\bar{x}(t))=V_{z}+V_{\theta}, \\
\bar{x}=\left[z(t), \widetilde{\theta}^{\top}\right]^{\top}, \\
V_{\theta}=\left(\frac{1}{2}\right) \tilde{\theta}^{\top} \Gamma^{-1} \widetilde{\theta},
\end{gathered}
$$

where $V_{z}$ is defined in (18), $\tilde{\theta}$ in (38), and $z$ in (12), and $\Gamma$ is a $3 \times 3$ diagonal matrix whose diagonal entries are user-defined positive constants. Note that according to the Lyapunov-like function definition and (48) and (51), the matrix $\Gamma$ must be definite positive; this is fulfilled if $\Gamma$ is a diagonal matrix whose entries are positive. The states of the closed loop system are $z$ and $\tilde{\theta}$. For the sake of easier understanding, the Lyapunov-like function $V(\bar{x}(t))(46)$ is rewritten in terms of $z$ and $\tilde{\theta}$, using expression (18):

$$
\begin{aligned}
V(\bar{x}(t))=V_{z}+\left(\frac{1}{2}\right) \tilde{\theta} \Gamma^{-1} \tilde{\theta}, \\
V_{z}(z)= \begin{cases}\left(\frac{1}{2}\right)\left(z-C_{b z}\right)^{2} & \text { if } z \geq C_{b z} \\
0 & \text { if } z \in\left(-C_{b z}, C_{b z}\right) \\
\left(\frac{1}{2}\right)\left(z+C_{b z}\right)^{2} & \text { if } z \leq-C_{b z} .\end{cases}
\end{aligned}
$$

Differentiating $V(\bar{x}(t))$ with respect to time and using (48) yield

$$
\begin{aligned}
\dot{V} & =\dot{V}_{z}+\dot{V}_{\theta} \\
\dot{V}_{\theta} & =\widetilde{\theta}^{\top} \Gamma^{-1} \dot{\widetilde{\theta}}=\widetilde{\theta}^{\top} \Gamma^{-1} \dot{\hat{\theta}},
\end{aligned}
$$

Substituting (45) and (51) into (50) yields

$$
\dot{V} \leq-c_{1} g_{z}^{2}-\varphi^{\top} \tilde{\theta}\left|g_{z}\right|+\widetilde{\theta}^{\top} \Gamma^{-1} \dot{\hat{\theta}}
$$

3.6. Step 6. In this step the updating law is formulated. Equation (52) can be rewritten as

$$
\dot{V} \leq-c_{1} g_{z}^{2}+\widetilde{\theta}^{\top}\left(-\varphi\left|g_{z}\right|+\Gamma^{-1} \dot{\hat{\theta}}\right)
$$

The update law is chosen such that the term $\tilde{\theta}^{\top} \varphi\left|g_{z}\right|$ is canceled:

$$
\dot{\hat{\theta}}=\Gamma \varphi\left|g_{z}\right|
$$

where $g_{z}$ is defined in (24) and $\Gamma$ is a $3 \times 3$ diagonal matrix whose diagonal elements are user-defined positive constants. Substituting (54) into (53) yields

$$
\dot{V} \leq-c_{1} g_{z}^{2} \leq 0
$$

The above expression fulfills properties (TDPi), (TDPii), and (TDPiii) mentioned in Step 2. Therefore, the observer error $z$ converges to a residual set whose size is $C_{b z}$, as will be proven in Section 4.

3.7. Step 7. In this step the control law is formulated based on the observer equation (44) $(\widehat{y})$ and the desired output dynamics $(10)\left(y_{d}\right)$, such that the difference $\widehat{y}-y_{d}$ converges asymptotically to zero when the control input does not get saturated. Let

$$
x=\widehat{y}-y_{d},
$$

where $\hat{y}$ is provided by (44). Differentiating with respect to time and using (44) yield

$$
\dot{x}=\dot{\hat{y}}-\dot{y}_{d}=\varphi^{\top} \hat{\theta} \mathrm{sat}_{z}+\bar{b} u+c_{1} g_{z}-\dot{y}_{d} .
$$

The input rule for the case of no saturation is based on the input-output linearization method [28], such that $x$ converges exponentially to zero. Such input rule cancels the nonlinear terms $c_{1} g_{z}, \varphi^{\top} \hat{\theta} s_{z}$, and $-\dot{y}_{d}$ and introduces the nonlinear term $-c_{2} x$ :

$$
u_{o}=\frac{1}{\bar{b}}\left(-c_{1} g_{z}-\varphi^{\top} \hat{\theta} \mathrm{sat}_{z}+\dot{y}_{d}-c_{2} x\right) \text {, }
$$

where $\bar{b}$ is defined in (27). The final control law involves the saturation limits $u_{\min }, u_{\max }$ :

$$
u= \begin{cases}u_{o} & \text { if } u_{o} \in\left[u_{\min }, u_{\max }\right] \\ u_{\max } & \text { if } u_{o}>u_{\max } \\ u_{\min } & \text { if } u_{o}<u_{\min }\end{cases}
$$

where $u_{\min }$ and $u_{\max }$ are the extreme values of the control input $D$ defined in Characteristic 4 and $c_{2}$ is a positive constant defined by the user. The observer mechanism (44), the update law (54), and the control law given by (59) and (58) achieve the convergence of $z$ to the residual set $\Omega_{z}=\{z$ : $\left.|z| \leq C_{b z}\right\}$, despite input saturation events, as will be proven in Section 4. 
Remark 5. When input saturation occurs, the bioreactor is in open loop operation, and it is difficult to guarantee the expected convergence of the tracking error. To the author's knowledge, the expected convergence of the tracking error under input saturation events has not been guaranteed for bioreactors. Nevertheless, as can be noticed from numerical simulation and experimental results shown in $[16,22]$, the input saturation events only occur for some lapses of time. Therefore, in the present work the convergence of the tracking error to a residual set of user-defined size is proven for the time lapses when input saturation does not occur.

The value of constant $C_{b z}$ is defined as the following, such that the tracking error $e$ converges to $\Omega_{e}=\left\{e:|e| \leq C_{b z}\right\}$, as expected in control goal (CGii) of Section 2.2. Recall that $z$ converges to $\Omega_{z}=\left\{z:|z| \leq C_{b z}\right\}$. If the control input $u_{o}$ defined in (58) fulfills $u_{o} \in\left[\begin{array}{ll}u_{\min } & u_{\max }\end{array}\right] \forall t \geq t_{o}$, then the signal $x$ defined in (56) converges to zero, and the tracking error $e$ converges to the residual set $\Omega_{e}=\left\{e:|e| \leq C_{b z}\right\}$, as is proven in Section 4. According to the control goal (CGii) and (11), the size of the residual set $\Omega_{e}$ should be $C_{b e}$. Therefore, the positive constant $C_{b z}$ is defined as

$$
C_{b z}=C_{b e}
$$

This completes the controller design.

Remark 6. The developed controller involves the control law (58)-(59), the update law (54), and the observer (44). The parameters necessary to implement it are (i) the nominal value $\bar{S}_{2}^{\text {in }}$ which satisfies $\bar{S}_{2}^{\text {in }}-y_{m}>0$ according to Characteristic 6 , (ii) the signals that depend on the states of the plant model and controller, namely, $y_{d}(10), z(12), g_{z}(24), \bar{b}(27)$, $\varphi$ (34), sat $_{z}$ (42), and $x$ (56), (iii) the user-defined constants, namely, $C_{b e}, c_{1}, c_{2}, \gamma_{1}, \gamma_{2}$, and $\gamma_{3}$, being $\gamma_{1}, \gamma_{2}$, and $\gamma_{3}$ the diagonal entries of $\Gamma$, and (iv) the constant $C_{b z}$ (60). The user-defined parameters, $\gamma_{1}, \gamma_{2}, \gamma_{3}, c_{1}, c_{2}$, and $a_{m}$ should be chosen according to simulation results so as to obtain desired evolution of the control input $u$, updated parameter vector $\widehat{\theta}$, and observer state $\widehat{y}$.

Remark 7. The control law (58)-(59), the update law (54), and the observer (44) depend on the observer error $z$, instead of the tracking error $e$. This is due to the fact that the quadratic form $V_{z}(18)$ is defined in terms of $z$ instead of $e$, and the Lyapunov-like function $V(46)$ is defined in terms of $V_{z}$.

Remark 8. The developed controller has two important features. First, the only required model parameter is $\bar{S}_{2}^{\text {in }}$; other upper or lower bounds of parameters of models (1) to (4) are not required as can be noticed from (44), (54), and (58)-(59). Indeed, the upper bounds $\bar{\kappa}_{1}, \bar{\kappa}_{2}$, and $\bar{\kappa}_{3}$ are not used. This implies less modeling effort. Second, it does not involve discontinuous signals. Indeed, it uses the continuous signal sat ${ }_{z}$, instead of $\operatorname{sgn}\left(g_{z}\right)$, so that the vector field of the closed loop system is locally Lipschitz continuous. In turn, this implies that input and state chattering are avoided, and trajectory unicity is guaranteed, according to [27]. Therefore,

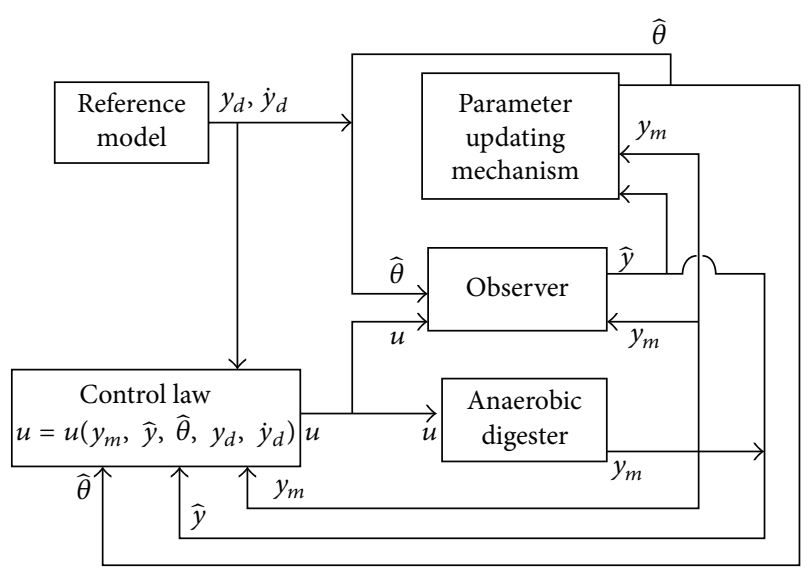

FIGURE 1: Schematic diagram of the developed controller, in terms of the basic signals $y_{m}, \hat{y}, \hat{\theta}, y_{d}$, and $\dot{y}_{d}$.

the benefits Bf 1.1 and $\mathrm{Bf} 1.4$ mentioned in introduction are achieved.

Remark 9. The signals and the corresponding equations required to compute the control law (58)-(59) are $g_{\mathrm{z}}(24), \hat{\theta}$ (54), sat $_{z}$ (42), $\varphi(34), y_{d}(10)$, and $x$ (56). After analyzing these expressions and signal $z$ defined in $z$ (12), it is concluded that (i) the control law (58)-(59) depends on the basic signals $y_{m}, \hat{y}, \hat{\theta}, y_{d}$, and $\dot{y}_{d}$, (ii) the observer mechanism (44) depends on signals $y_{m}, \widehat{\theta}$, and $u$; therefore, it depends on the basic signals $y_{m}, \widehat{\theta}, y_{d}$, and $\dot{y}_{d}$, and (iii) the parameter updating mechanism (54) depends on the basic signals $y_{m}$, $\widehat{y}$. Figure 1 shows a schematic diagram of the developed controller, in terms of the basic signals.

\section{Stability Analysis}

In this section it is proven that the closed loop signals $z$ and $\widetilde{\theta}$ are bounded, and that the observer error $z$ converges to a residual set of predefined size despite input saturation. In addition, it is proven that the tracking error $e$ converges asymptotically to a residual set of user-defined size for the time lapses during which the control input does not get saturated. Recall that in [16] the convergence of the observer error to a residual set of small size is guaranteed if the constant user-defined parameters of the control and observer mechanisms are properly defined. Nevertheless, such size is unknown, so that the correct choices of the constant userdefined parameters are unknown. It is difficult to prove the convergence of the tracking error for the case of input saturation, because the system gets in open loop.

The stability analysis of the present section is based on the Lyapunov-like function method presented in [32-34], which will be applied to (55). The bounded nature of the signals $z$ and $\tilde{\theta}$ and the Barbalat's Lemma allow us to prove the convergence of the observer error $z$. Moreover, the bounded nature of $\tilde{\theta}$ implies that $\widehat{\theta}$ is also bounded, so that $\widehat{\theta}$ does 
not experience drifting, and consequently, the control input $u$ does not exhibit excessive magnitude.

Theorem 10 (boundedness of the closed loop signals). Consider (i) the plant model (1) to (4), subject to Characteristics 1 to 6 and Assumption 1; (ii) the controller, which involves the control law (58)-(59), the update law (54), and the observer mechanism (44); (iii) the signals that depend on the states of the plant model and controller, namely, $y_{d}(10), z(12), g_{z}(24)$, $\bar{b}$ (27), $\varphi$ (34), sat s $_{z}$ (42), $x$ (56); (iv) the user-defined constants, namely, $C_{b e}, c_{1}, c_{2}, \gamma_{1}, \gamma_{2}$, and $\gamma_{3}$, being $\gamma_{1}, \gamma_{2}$, and $\gamma_{3}$ the diagonal entries of $\Gamma$; and $(v)$ the definition of $C_{b z}(60)$. If this controller is employed to define the value of the control input $u$, then (i) the signals $\hat{y}, \hat{\theta}$, and $u$ remain bounded and (ii) the transient $z$ is upper bounded by a positive constant:

$$
\begin{aligned}
&|z| \leq C_{b z} \\
&+\sqrt{2}\left\{V_{z o}+\left(\frac{1}{2}\right)\right. \\
& \quad \times\left(\gamma_{1}^{-1}\left(\widehat{\theta}_{1}\left(t_{o}\right)-\theta_{1}\right)^{2}+\gamma_{2}^{-1}\left(\widehat{\theta}_{2}\left(t_{o}\right)-\theta_{2}\right)^{2}\right. \\
&\left.\left.\quad+\gamma_{3}^{-1}\left(\widehat{\theta}_{3}\left(t_{o}\right)-\theta_{3}\right)^{2}\right)\right\}^{1 / 2}
\end{aligned}
$$

where

$$
\begin{aligned}
V_{z o} & = \begin{cases}\left(\frac{1}{2}\right)\left(z\left(t_{o}\right)-C_{b z}\right)^{2} & \text { if } z\left(t_{o}\right) \geq C_{b z} \\
0 & \text { if } z\left(t_{o}\right) \in\left(-C_{b z}, C_{b z}\right) \\
\left(\frac{1}{2}\right)\left(z\left(t_{o}\right)+C_{b z}\right)^{2} & \text { if } z\left(t_{o}\right) \leq-C_{b z}\end{cases} \\
z\left(t_{o}\right) & =y_{m}\left(t_{o}\right)-\hat{y}\left(t_{o}\right) .
\end{aligned}
$$

Proof. Recall that $z$ and $\widetilde{\theta}$ were defined in (12) and (38), respectively. From (55) it follows that $V(\bar{x}(t)) \leq V\left(\bar{x}\left(t_{o}\right)\right)$, so that $V(\bar{x}(t)) \in L_{\infty}$. Therefore, from (46) it follows that $V_{\theta} \in L_{\infty}$ and $V_{z} \in L_{\infty}$; from (48), it follows that $\widetilde{\theta} \in L_{\infty}$; from (38), it follows that $\widehat{\theta} \in L_{\infty}$; from (18), it follows that $z \in L_{\infty}$; from (24), it follows that $g_{z} \in L_{\infty}$; from Lemma A.5 and Assumption 1 it follows that $y=S_{2} \in L_{\infty}$ and $y_{m}=S_{2 m} \in$ $L_{\infty}$. The properties $z \in L_{\infty}, y_{m} \in L_{\infty}$, and definition (12) lead to $\hat{y}=y_{m}-z$ and $\hat{y} \in L_{\infty}$. This completes the proof for the first part of Theorem 10. Arranging and integrating (55) yield

$$
V(\bar{x}(t))+c_{1} \int_{t_{o}}^{t} g_{z}^{2} d \tau \leq V\left(\bar{x}\left(t_{o}\right)\right)
$$

where $V\left(\bar{x}\left(t_{o}\right)\right)$ is the initial value of $V(\bar{x}(t))$. Evaluating $V(\bar{x}(t))(18), V_{z}(46)$, and $V_{\theta}$ (48) for $t=t_{o}$ yields

$$
V\left(\bar{x}\left(t_{o}\right)\right)=V_{z o}+\left(\frac{1}{2}\right)\left(\widehat{\theta}\left(t_{o}\right)-\theta\right)^{\top} \Gamma^{-1}\left(\widehat{\theta}\left(t_{o}\right)-\theta\right)^{\top},
$$

$$
\begin{gathered}
V_{z o}= \begin{cases}\left(\frac{1}{2}\right)\left(z\left(t_{o}\right)-C_{b z}\right)^{2} & \text { if } z\left(t_{o}\right) \geq C_{b z} \\
0 & \text { if } z\left(t_{o}\right) \in\left(-C_{b z}, C_{b z}\right) \\
\left(\frac{1}{2}\right)\left(z\left(t_{o}\right)+C_{b z}\right)^{2} & \text { if } z\left(t_{o}\right) \leq-C_{b z},\end{cases} \\
z\left(t_{o}\right)=y_{m}\left(t_{o}\right)-\hat{y}\left(t_{o}\right) .
\end{gathered}
$$

From (46), (64), and (65) it follows that

$$
\begin{aligned}
V_{z} & +V_{\theta}+c_{1} \int_{t_{o}}^{t} g_{z}^{2} d \tau \\
& \leq V_{z o}+\left(\frac{1}{2}\right)\left(\widehat{\theta}\left(t_{o}\right)-\theta\right)^{\top} \Gamma^{-1}\left(\widehat{\theta}\left(t_{o}\right)-\theta\right)^{\top}, \\
V_{z} & \leq V_{z o}+\left(\frac{1}{2}\right)\left(\widehat{\theta}\left(t_{o}\right)-\theta\right)^{\top} \Gamma^{-1}\left(\widehat{\theta}\left(t_{o}\right)-\theta\right)^{\top} .
\end{aligned}
$$

Incorporating the property (20) yields

$$
\begin{aligned}
&|z| \leq C_{b z} \\
&+\sqrt{2}\left\{V_{z o}+\left(\frac{1}{2}\right)\right. \\
& \quad \times\left(\gamma_{1}^{-1}\left(\widehat{\theta}_{1}\left(t_{o}\right)-\theta_{1}\right)^{2}+\gamma_{2}^{-1}\left(\widehat{\theta}_{2}\left(t_{o}\right)-\theta_{2}\right)^{2}\right. \\
&\left.\left.\quad+\gamma_{3}^{-1}\left(\widehat{\theta}_{3}\left(t_{o}\right)-\theta_{3}\right)^{2}\right)\right\}^{1 / 2},
\end{aligned}
$$

where $\widehat{\theta}_{1}\left(t_{o}\right), \widehat{\theta}_{2}\left(t_{o}\right)$, and $\widehat{\theta}_{3}\left(t_{o}\right)$ are the first, second, and third entries of the vector $\widehat{\theta}\left(t_{0}\right)$. End of proof.

Remark 11. Theorem 10 indicates that the transient behavior of the observer error $z$ is bounded by a constant limit that depends on (i) the user-defined initial values $\widehat{\theta}_{1}\left(t_{o}\right), \widehat{\theta}_{2}\left(t_{o}\right)$, and $\widehat{\theta}_{3}\left(t_{o}\right)$, (ii) the initial value $z\left(t_{o}\right)=y_{m}\left(t_{o}\right)-\widehat{y}\left(t_{o}\right)$, and (iii) the user-defined constants $\gamma_{1}, \gamma_{2}, \gamma_{3}$, and $C_{b z}$. Therefore, the transient value of $z$ can be constrained to lower values by choosing large values of $\gamma_{1}, \gamma_{2}$, and $\gamma_{3}$ and low values of $C_{b z}$, and choosing $y_{m}\left(t_{o}\right)$ such that $y_{m}\left(t_{o}\right)-\hat{y}\left(t_{o}\right)$ is small.

In control designs that involve observers (see $[15,16])$, the goal of the observer is that the estimated output converges towards the real output with a small threshold $C_{b z}$. In the present work such goal is assumed in the form of goal (CGi). The proof of the observer error convergence is based on the application of the Barbalat's Lemma to expression (55). The result is that $g_{z}$ converges to zero and $z$ converges to the residual set $\Omega_{z}=\left\{z:|z| \leq C_{b z}\right\}$. This implies the convergence of the tracking error towards a residual set, whose size is user-defined. Notice that the asymptotic convergence of the observer error is achieved despite the input saturation and despite the fact that the exact values and upper bounds of plant and noise model parameters are assumed unknown.

Theorem 12 (Convergence of the observer error). Consider (i) the plant model (1) to (4), subject to Characteristics 1 to 6 and 
Assumption 1: (ii) the controller, which involves the control law (58)-(59), the update law (54), and the observer mechanism (44), (iii) the signals that depend on the states of the plant model and controller, namely, $y_{d}(10), z$ (12), $g_{z}$ (24), $\bar{b}$ (27), $\varphi$ (34), sat $z_{z}$ (42), and $x$ (56), (iv) the user-defined constants, namely, $C_{b e}, c_{1}, c_{2}, \gamma_{1}, \gamma_{2}$, and $\gamma_{3}$, being $\gamma_{1}, \gamma_{2}$, and $\gamma_{3}$ the diagonal entries of the matrix $\Gamma$, and $(v)$ the definition of $C_{b z}$ (60). If this controller is employed to define the value of the control input $u$, then $z$ converges asymptotically to $\Omega_{z}$, where $\Omega_{z}=\left\{z:|z| \leq C_{b z}\right\}$ and $C_{b z}=C_{b e}$.

Proof. From (64) it follows that $g_{z}^{2} \in L_{1}$. It is necessary to prove that $g_{z}^{2} \in L_{\infty}$ and $d\left(g_{z}^{2}\right) / d t \in L_{\infty}$ to apply the Barbalat's Lemma. Since $g_{z} \in L_{\infty}$, then $g_{z}^{2} \in L_{\infty}$. Signals $V_{z}$ in (18) and $\partial V_{z} / \partial z$ in (24) lead to $V_{z}=(1 / 2) g_{z}^{2}, g_{z}^{2}=2 V_{z}$. Differentiating with respect to time yields

$$
\frac{d g_{z}^{2}}{d t}=2 \dot{V}_{z}=2 g_{z} \dot{z}
$$

It is necessary to prove that $\dot{z} \in L_{\infty}$. From Lemma A.5 and Assumption 1 it follows that $y \in L_{\infty}$ and $y_{m} \in L_{\infty}$. Since $y_{m} \in L_{\infty}, \hat{\theta} \in L_{\infty}$, and $g_{z} \in L_{\infty}$, it follows that $\varphi \in L_{\infty}$ (34) and $\dot{\hat{y}} \in L_{\infty}$ (44). Since $\dot{\hat{y}} \in L_{\infty}, \dot{a}_{1} \in L_{\infty}, a_{o} \in L_{\infty}$, $a_{1} \in L_{\infty}, \dot{a}_{2} \in L_{\infty}, b \in L_{\infty}, u \in L_{\infty}, y \in L_{\infty}$, it follows that $\dot{z} \in L_{\infty}$ (15). Therefore, $d g_{z}^{2} / d t \in L_{\infty}$ (71). So far, it has been proved that $g_{z}^{2} \in L_{1}, g_{z}^{2} \in L_{\infty}, d\left(g_{z}^{2}\right) / d t \in L_{\infty}$. Applying the Barbalat's Lemma, cf. [36], yields: $\lim _{t \rightarrow \infty} g_{z}^{2}=0$. Thus, $z$ (24) converges asymptotically to $\Omega_{z}$, where $\Omega_{z}=\left\{z:|z| \leq C_{b z}\right\}$. End of proof

It is important to ensure that the measured output $y_{m}$ converges towards the desired output $y_{d}$, with some userdefined threshold $C_{b e}$. Therefore, it will be proven that the tracking error $e$ converges asymptotically towards the residual set $\Omega_{e}=\left\{e:|e| \leq C_{b e}\right\}$ when the control input does not get saturated. To that end, the convergence of $z$ towards $\Omega_{z}$ is taken into account and the relationship between $z, x$, and $e$ is established. The drawback of such proof is that it is only valid when the control input is not saturated.

Theorem 13 (convergence of the tracking error). Consider (i) the plant model (1) to (4), subject to Characteristics 1 to 6 and Assumption 1: (ii) the controller, which involves the control law (58)-(59), the update law (54), and the observer mechanism (44), (iii) the signals that depend on the states of the plant model and controller, namely, $y_{d}(10), z(12), g_{z}(24), \bar{b}(27), \varphi(34)$, sat $_{z}$ (42), and $x$ (56), (iv) the user-defined constants, namely, $C_{b e}, c_{1}, c_{2}, \gamma_{1}, \gamma_{2}$, and $\gamma_{3}$, being $\gamma_{1}, \gamma_{2}$, and $\gamma_{3}$ the diagonal entries of matrix $\Gamma$, and $(v)$ the definition of $C_{b z}(60)$. If this controller is employed to define the value of the control input $u$ and the computed signal control $u_{o} \in\left[u_{\min }, u_{\max }\right]$, then the tracking error e converges asymptotically to $\Omega_{e}$, where $\Omega_{e}=\{e:|e| \leq$ $\left.C_{b e}\right\}$.

Proof. Setting $u=u_{o}$ and replacing (58) in (57) yield

$$
\dot{x}=-c_{2} x
$$

This implies that $x$ converges asymptotically to zero. The tracking error $e$ can be expressed in terms of $z$ and $x$. Signals $e$ in (9), $z$ in (12), and $x$ in (56) lead to

$$
e=y_{m}-y_{d}=\left(y_{m}-\widehat{y}\right)+\left(\widehat{y}-y_{d}\right)=z+x
$$

Since $x$ converges asymptotically to zero, and $z$ converges asymptotically to $\Omega_{z}=\left\{z:|z| \leq C_{b z}\right\}$, where $C_{b z}=C_{b e}$, it follows that $e$ converges asymptotically to $\Omega_{e}=\left\{e:|e| \leq C_{b e}\right\}$. End of proof.

Remark 14. If the control input gets saturated, then (i) the closed loop signals $z, \widetilde{\theta}$, and $\hat{y}$ remain bounded, as stated in Theorem 10, (ii) the observer error $z$ converges asymptotically to a residual set of size $C_{b z}$ with $C_{b z}=C_{b e}$, as stated in Theorem 12, and (iii) the convergence of the tracking error to a residual set of predefined size is not guaranteed. Indeed, when the input gets saturated, that is, $u_{o}>u_{\max }$ or $u_{o}<u_{\min }$, the expression (72) is not valid, so that the convergence of the tracking error is not guaranteed.

The above stability analysis shows that benefits Bf 1.2 , $\mathrm{Bf} 1.3$, Bf 1.5, and $\mathrm{Bf} 1.6$ mentioned in introduction are accomplished.

\section{Simulation Example}

In this example, the plant (1) to (4), subject to Characteristics 1 to 6 and Assumption 1, is considered. The values of the yield coefficients, kinetic parameters, and proportion of biomass not attached to the reactor appearing in (1) to (5) are taken from [12]. The controller mechanism and the corresponding parameters are stated in Remark 6. The considered scenario is the same as that in [32] and consequently similar to that in [16], that is, (i) the influent concentrations of VFA and COD are varying, (ii) output measurement is noisy, (iii) the command signal $r$ involves changes larger than $4 \mathrm{mmol} / \mathrm{L}$, and (iv) the control input gets saturated. The corresponding values are similar to those in [16], in order to compare the performance of the controller and the observer. The values of some parameters are

$$
\begin{gathered}
S_{1}^{\text {in }}=9\left(1+0.3 \sin \left(\frac{2 \pi}{4.2} t\right)\right)[\mathrm{g} / \mathrm{L}], \\
S_{2}^{\text {in }}=100\left(1+0.3 \sin \left(\frac{2 \pi}{3.8} t\right)\right)[\mathrm{mmol} / \mathrm{L}], \\
D \in\left[\begin{array}{ll}
0 & 1.2
\end{array}\right] \mathrm{day}^{-1}, \\
a_{1}=1, \quad a_{2}=2 \sin \left(\frac{2 \pi}{0.42} t\right),
\end{gathered}
$$

where $a_{1}$ and $a_{2}$ are the parameters considered in the noise model (7). Recall that the values of $S_{1}^{\text {in }}, S_{2}^{\text {in }}, a_{1}$, and $a_{2}$ are not used by the controller, neither by the control law, nor the update law nor the observer mechanism. The controlled is started at $t=6.25$ days. From $[12,14]$ it follows that $S_{2}$ is always lower than $S_{2}^{\text {in }}$. Since $S_{2}^{\text {in }}$ follows the trajectory (75), $S_{2}$ 
satisfies $S_{2}<100 \mathrm{mmol} / \mathrm{L}$, and thus the value $100 \mathrm{mmol} / \mathrm{L}$ is chosen for $\bar{S}_{2}$. The values of the controller parameters are

$$
\begin{gathered}
\bar{S}_{2}^{\text {in }}=100 \mathrm{mmol} / \mathrm{L}, \\
u_{\min }=0, \quad u_{\max }=1.2 \text { day }^{-1}, \\
r=15 \quad \text { if } 6.25 \leq t<8.33 \text { day, } \\
r=10 \quad \text { if } 8.33 \leq t<16.7 \text { day, } \\
r=18 \quad \text { if } 16.7 \leq t<29.2 \text { day, } \\
r=10 \quad \text { if } 29.2 \leq t<33 \text { day, } \\
C_{b e}=0.3, \quad a_{m}=6, \quad c_{1}=1, \\
c_{2}=10, \quad \gamma_{1}=10, \quad \gamma_{2}=10, \quad \gamma_{3}=10,
\end{gathered}
$$

where $\gamma_{1}, \gamma_{2}$, and $\gamma_{3}$ are the diagonal entries of the matrix $\Gamma$ appearing in the update law (54); the parameters $r$ and $a_{m}$ correspond to the reference model (10); $\bar{S}_{2}$ in required to be known and satisfies Characteristic 6 . The results are shown in Figures 2 and 3, with Figure 3 being a zooming of Figure 2.

Figure 3 indicates the following: (i) the estimated output $\hat{y}$ converges towards the measured output $y_{m}$, such that the observer error $z$ converges to the residual set $\Omega_{z}$ and remains therein after the time instant of 14.5 days, despite the effect of the varying behavior of $S_{1}^{\text {in }}$ and $S_{2}^{\text {in }}$, the output measurement noise and the control input saturation, (ii) the output measurement $y_{m}$ tracks the desired output $y_{d}$, so that the tracking error $e$ converges to the residual set $\Omega_{e}$, but such convergence is degraded when the control input gets saturated, (iii) the output measurement $y_{m}$ exhibits sustained oscillations due to measurement noise and oscillations in $S_{1}^{\text {in }}$ and $S_{2}^{\text {in }}$, (iv) the control input $u$ exhibits high and low frequency oscillations due to the measurement noise and the oscillations in $S_{1}^{\text {in }}$ and $S_{2}^{\text {in }}$, and (v) the control input $u$ gets saturated during some intervals which is owed to the enormous magnitude of the disturbances in $S_{1}^{\text {in }}$ and $S_{2}^{\text {in }}$ and the measurement noise (close to 30\%).

\section{Discussion and Conclusions}

In this work a controller for an anaerobic digester, with output measurement noise and constrained input, has been designed using the Lyapunov-like function method. The Characteristics 1 to 6 and Assumption 1 were taken into account in the control design and the stability analysis. The benefits Bf 1.1 to Bf 1.6 are achieved, and the main ones can be recalled as follows: (i) the exact values of the parameters of both plant model and noise model are not required to be known; (ii) although a nominal value of $S_{2}^{\text {in }}$, denoted by $\bar{S}_{2}^{\text {in }}$, is required to be known, other upper or lower bounds of the parameters of the plant and noise models are not required to be known; (iii) discontinuous signals are avoided in the control law, the update law, and the observer mechanism, so that undesired state chattering and input chattering are avoided; (iv) the boundedness of the closed loop signals is guaranteed, so that parameter drifting is avoided; (v) the observer error converges to a residual set of user-defined size, and such size is not degraded, neither by the uncertainty on the model parameters nor by the input saturation; and (vi) it is guaranteed that the tracking error converges to the expected residual when the control input does not get saturated.

The time-varying behavior of the model parameters implies that (i) the system is nonautonomous and (ii) the coordinates of the equilibrium points vary with time. Therefore, it is difficult to guarantee the local asymptotic stability by means of the indirect Lyapunov method. Nevertheless, the developed boundedness analysis indicates that the concentrations of acidogenic biomass, methanogenic biomass, COD, and VFA are bounded for nonnegative values of the dilution rate, despite large values of the dilution rate, regardless of the control law, the update law, and the observer mechanism used and regardless of the structure of the specific growth rates $\mu_{1}$ and $\mu_{2}$. In fact, the current structures of specific growth rates, for instance Monod and Haldane growth rates, are usually bounded, even if the substrate concentrations were not bounded. The bounded nature of the digester concentrations allows us to tackle the lack of knowledge on the reaction rate terms and to prove that the closed loop signals are bounded.

The state observer was used in order to handle the effect of input saturation, not to estimate any unknown state. The control law (58)-(59) involves an integral action appearing in the updated parameter $\hat{\theta}$, as can be noticed from (54). Nevertheless, excessive integral action caused by input saturation does not occur. In addition, the convergence of the observer error $z$ to $\Omega_{z}$ and the size of this residual set are not altered by the input saturation (see Section 4). It is difficult to establish the influence of input saturation on the convergence and transient behavior of the tracking error $e$ due to the nonlinear nature of the plant model and closed loop system.

The numerical simulation example indicates the following: (i) the reference model (10) leads to smooth behavior of $y_{d}$ when the reference signal $r$ exhibits step changes, thus contributing to diminish the input saturation periods; (ii) the tracking error converges to a residual set of predefined size when the control input is not saturated; and (iii) the convergence of the tracking error is degraded when the control input gets saturated.

\section{Appendices}

\section{A. Proof of Lemma 4}

The upper constant bounds of the digester states and reaction rate terms are established, in order to allow the controller design and its stability analysis.

The bounded nature of biological concentrations can be established by using the indirect Lyapunov method which is based on the Jacobian matrix or other techniques as geometric control theory and state transformations, as it is discussed in what follows. In $[19,22,37,38]$ the stability of the digester presented in [14] was analyzed by means of the indirect Lyapunov method. The equilibrium point 


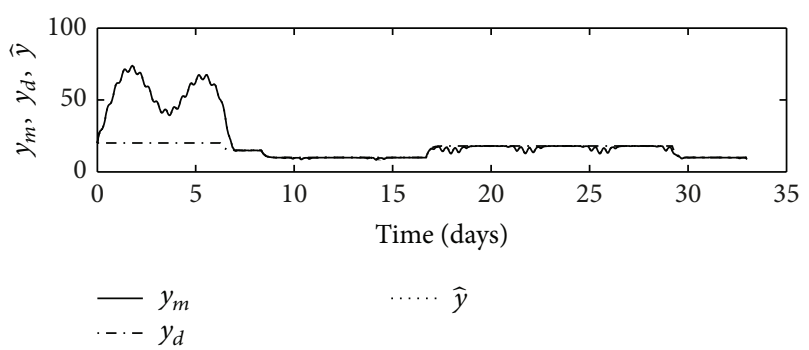

(a)

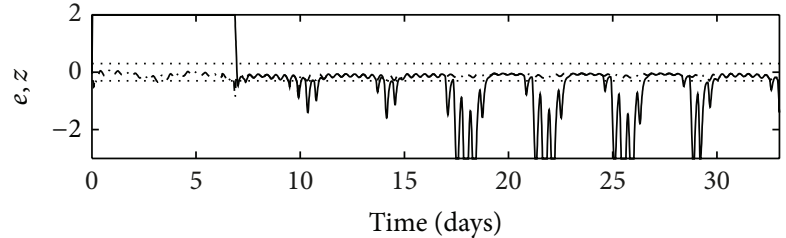

$-e$

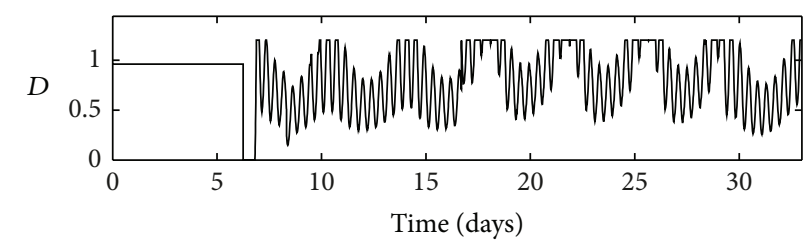

(b)

(c)

Figure 2: Example 1: (a) output measurement $y_{m}[\mathrm{mmol} / \mathrm{L}]$ (solid line), desired output $y_{d}$ (dash-dot line), and estimated output $\widehat{y}$ (dotted line); (b) tracking error $e$ (solid line) and observer error $z$ (dash-dot line); (c) control input $D$ [day $\left.{ }^{-1}\right]$.

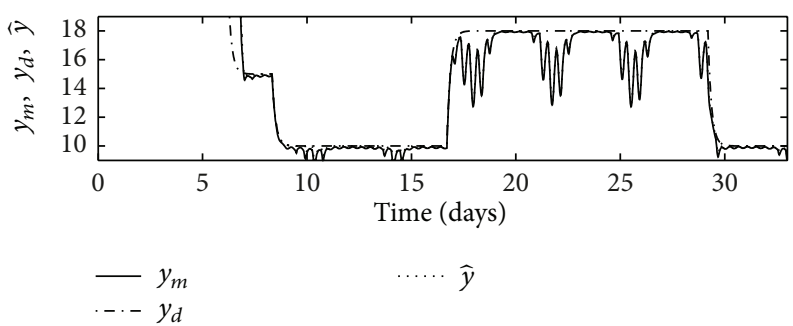

(a)

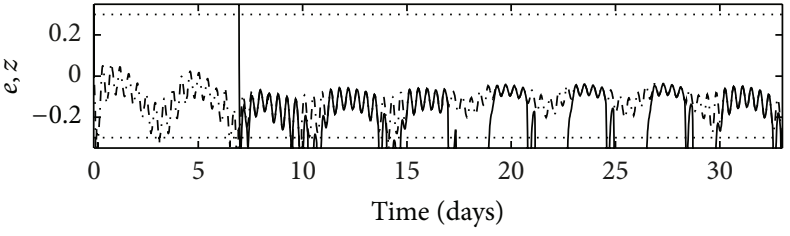

$-e$

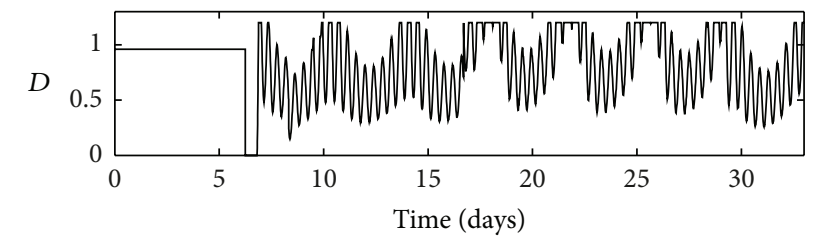

(c)

Figure 3: Example 1, zoomed view: (a) output measurement $y_{m}[\mathrm{mmol} / \mathrm{L}]$ (solid line), desired output $y_{d}$ (dash-dot line), and estimated output $\hat{y}$ (dotted line); (b) tracking error $e$ (solid line) and observer error $z$ (dash-dot line); (c) control input $D$ [day ${ }^{-1}$ ].

concerning the normal open loop operation of the digester is asymptotically stable, under nonnegative dilution rate. Nevertheless, the stoichiometric coefficients, the proportion of biomass not attached to the reactor and feed concentrations are assumed constant. This is in contrast to the fact that plant parameters, mainly the feed concentrations, exhibit significative time dependent variation, as mentioned in $[12,39]$. In $[16,40]$ the digester model is rewritten in canonical form and the stability of the internal dynamics is proven. Nevertheless, yield coefficients and feed concentrations are assumed constant in the development of the state transformation. In [19], constant upper bounds are established for the concentrations of acidogenic biomass and chemical oxygen demand (COD), under nonnegative dilution rate. To that end, a state transformation is carried out by defining a linear combination of acidogenic biomass and COD concentrations. Nevertheless, the yield coefficients and the proportion of biomass not attached to reactor are assumed constant in order to allow the development of the state transformation.

By contrast, in the present work it is established that the concentrations of acidogenic biomass, methanogenic biomass, chemical oxygen demand (COD), and VFA are bounded under the following conditions: (i) time-varying but nonnegative behavior of the dilution rate $D$ and (ii) time-varying but bounded behavior of the yield coefficients, 
proportion of biomass not attached to the reactor, kinetic parameters, and inflow concentrations.

The characteristics stated in Lemma 4 are established through several lemmas, in order to facilitate the understanding and the development of the procedure. The proofs involve several state transformations, with upper or lower constant bounds of plant model parameters as coefficients.

Lemma A.1. Consider the plant (1) to (4), subject to Characteristics 1 to 6 and Assumption 1. The concentrations of acidogenic biomass and COD, that is, $X_{1}$ and $S_{1}$, are upper bounded by positive constants as follows:

$$
\begin{gathered}
\quad S_{1} \leq \max \left\{\frac{S_{1 i \max }}{\min \left\{1, \alpha_{\min }\right\}}, S_{1}\left(t_{o}\right)+k_{1 \min } X_{1}\left(t_{o}\right)\right\} \\
X_{1} \\
\leq\left(\frac{1}{k_{1 \min }}\right) \max \left\{\frac{S_{1 i \max }}{\min \left\{1, \alpha_{\min }\right\}}, S_{1}\left(t_{o}\right)+k_{1 \min } X_{1}\left(t_{o}\right)\right\},
\end{gathered}
$$

where $S_{1 \text { max }}, \alpha_{\min }$, and $k_{1 \text { min }}$ are constant bounds established in Characteristic 3. The above statement does not depend on the control law, the update law, or the observer mechanism used.

Proof. Let

$$
Z_{1}=S_{1}+k_{1 \min } X_{1}
$$

where $k_{1 \text { min }}$ is the lower bound of $k_{1}$, as considered in Characteristic 3. Differentiating (A.2) with respect to time and using trajectories (1) and (3) yield

$$
\dot{Z}_{1}=\left(S_{1}^{\text {in }}-S_{1}\right) D-k_{1} \mu_{1} X_{1}+k_{1 \min } \mu_{1} X_{1}-\alpha D k_{1 \min } X_{1} .
$$

From Characteristics 3 and 2 and expression (5) it follows that $k_{1 \text { min }} \leq k_{1}, \mu_{1} \geq 0$, and $X_{1} \geq 0$. Therefore,

$$
\begin{gathered}
\mu_{1} X_{1} \geq 0, \\
-k_{1} \mu_{1} X_{1} \leq-k_{1 \min } \mu_{1} X_{1}-k_{1} \mu_{1} X_{1}+k_{1 \min } \mu_{1} X_{1} \leq 0 .
\end{gathered}
$$

Substituting (A.4) into (A.3) yields

$$
\dot{Z}_{1} \leq-S_{1} D-\alpha D k_{1 \min } X_{1}+S_{1}^{\text {in }} D .
$$

Characteristic 3 mentions that $\alpha \geq \alpha_{\min }, S_{1}^{\text {in }} \leq S_{1 i \max }$, so that

$$
\dot{Z}_{1} \leq-S_{1} D-\alpha_{\min } D k_{1 \min } X_{1}+S_{1 i \max } D .
$$

Using the inequalities $1 \geq \min \left\{1, \alpha_{\min }\right\}$ and $\alpha_{\min } \geq$ $\min \left\{1, \alpha_{\min }\right\}$ yields

$$
\begin{aligned}
\dot{Z}_{1} \leq & -\min \left\{1, \alpha_{\min }\right\} \\
& \times\left[S_{1}+k_{1 \min } X_{1}-\frac{S_{1 i \max }}{\min \left\{1, \alpha_{\min }\right\}}\right] D .
\end{aligned}
$$

Let

$$
\begin{aligned}
& \bar{Z}_{1}=Z_{1}-\frac{S_{1 i \max }}{\min \left\{1, \alpha_{\min }\right\}} \\
& \bar{Z}_{1}=S_{1}+k_{1 \min } X_{1}-\frac{S_{1 i \max }}{\min \left\{1, \alpha_{\min }\right\}},
\end{aligned}
$$

where $Z_{1}$ is defined in (A.2). Substituting (A.9) into (A.7) yields

$$
\dot{Z}_{1} \leq-\min \left\{1, \alpha_{\min }\right\} \bar{Z}_{1} D \text {. }
$$

Differentiating (A.8) with respect to time yields $\dot{\bar{Z}}_{1}=\dot{Z}_{1}$. Using (A.10) yields $\dot{\bar{Z}}_{1} \leq-\min \left\{1, \alpha_{\min }\right\} \bar{Z}_{1} D$. Using Lemma in [28] yields

$$
\bar{Z}_{1} \leq \bar{Z}_{1 o} e^{-\min \left\{1, \alpha_{\min }\right\} D\left(t-t_{o}\right)},
$$

where $\bar{Z}_{1 o}$ is the initial value of $\bar{Z}_{1}$ and can be obtained by evaluating (A.9) for $t=t_{o}$ :

$$
\bar{Z}_{1 o}=S_{1}\left(t_{o}\right)+k_{1 \min } X_{1}\left(t_{o}\right)-\frac{S_{1 i \max }}{\min \left\{1, \alpha_{\min }\right\}} .
$$

From (A.11) it follows that

$$
\bar{Z}_{1} \leq \max \left\{0, \bar{Z}_{1 o}\right\} .
$$

Substituting (A.9) and (A.12) into (A.13) yields

$$
\begin{aligned}
S_{1}+ & k_{1 \min } X_{1}-\frac{S_{1 i \max }}{\min \left\{1, \alpha_{\min }\right\}} \\
\leq & \max \left\{0, S_{1}\left(t_{o}\right)+k_{1 \min } X_{1}\left(t_{o}\right)-\frac{S_{1 i \max }}{\min \left\{1, \alpha_{\min }\right\}}\right\}, \\
S_{1}+ & k_{1 \min } X_{1} \\
\leq & \frac{S_{1 i \max }}{\min \left\{1, \alpha_{\min }\right\}} \\
& +\max \left\{0, S_{1}\left(t_{o}\right)+k_{1 \min } X_{1}\left(t_{o}\right)-\frac{S_{1 i \max }}{\min \left\{1, \alpha_{\min }\right\}}\right\}, \\
S_{1}+ & k_{1 \min } X_{1} \\
\leq & \max \left\{\frac{S_{1 i \max }}{\min \left\{1, \alpha_{\min }\right\}}, S_{1}\left(t_{o}\right)+k_{1 \min } X_{1}\left(t_{o}\right)\right\} .
\end{aligned}
$$

Notice that expression (A.14) is different to that of [19], because the time-varying behavior of the yield coefficients and proportion $\alpha$ have been taken into account. Expression (A.14) leads to

$$
\begin{aligned}
& S_{1} \leq \max \left\{\frac{S_{1 i \max }}{\min \left\{1, \alpha_{\min }\right\}}, S_{1}\left(t_{o}\right)+k_{1 \min } X_{1}\left(t_{o}\right)\right\}, \\
& X_{1} \\
& \leq\left(\frac{1}{k_{1 \min }}\right) \max \left\{\frac{S_{1 i \max }}{\min \left\{1, \alpha_{\min }\right\}}, S_{1}\left(t_{o}\right)+k_{1 \min } X_{1}\left(t_{o}\right)\right\},
\end{aligned}
$$


and these are the constant upper bounds for $X_{1}$ and $S_{1}$. End of proof.

Remark A.2. The expressions in Lemma A.1 indicate that $X_{1}$ and $S_{1}$ are bounded if the dilution rate $D$ is nonnegative, regardless of the control law, update law, and observer used. In addition, $X_{1}$ and $S_{1}$ are bounded for any structure of the specific growth rate used for $\mu_{1}$ that is continuous with respect to substrate concentration. The reason is that the concentrations $X_{1}$ and $S_{1}$ are bounded for nonnegative dilution rate $D$, as is concluded from the above proof.

Lemma A.3. Consider the plant (1) to (4), subject to Characteristics 1 to 6 and Assumption 1. The reaction rate term $k_{2} \mu_{1} X_{1}$ is bounded as follows: $k_{2} \mu_{1} X_{1} \leq \bar{\kappa}_{\mu_{1} X_{1}}$, where $\bar{\kappa}_{\mu_{1} X_{1}}$ is a positive constant defined as

$$
\begin{aligned}
\bar{\kappa}_{\mu_{1} X_{1}} \\
=\frac{k_{2 \max } \mu_{1 \max }}{k_{1 \min }} \\
\quad \times \frac{\left[\max \left\{S_{1 i \max } / \min \left\{1, \alpha_{\min }\right\}, S_{1}\left(t_{o}\right)+k_{1 \min } X_{1}\left(t_{o}\right)\right\}\right]^{2}}{K_{S 1}+\max \left\{S_{1 i \max } / \min \left\{1, \alpha_{\min }\right\}, S_{1}\left(t_{o}\right)+k_{1 \min } X_{1}\left(t_{o}\right)\right\}},
\end{aligned}
$$

where $k_{2 \max }, S_{1 i \max }, \alpha_{\min }$, and $k_{1 \min }$ are constant bounds established in Characteritic 3. This statement is independent of the control law, the update law, and observer mechanism used.

Proof. The upper bound for $k_{2} \mu_{1} X_{1}$ can be established by using (A.16) and (A.15). The function $\mu_{1}$ (5) is monotonically increasing with respect to $S_{1}$, as mentioned in [18]. Thus, the maximum value of $\mu_{1}$ is obtained by replacing the maximum value of $S_{1}$ (A.15) into $\mu_{1}$ (5):

$$
\begin{aligned}
& \mu_{1} \\
& \leq \mu_{1 \max } \frac{\max \left\{S_{1 i \max } / \min \left\{1, \alpha_{\min }\right\}, S_{1}\left(t_{o}\right)+k_{1 \min } X_{1}\left(t_{o}\right)\right\}}{K_{S 1}+\max \left\{S_{1 i \max } / \min \left\{1, \alpha_{\min }\right\}, S_{1}\left(t_{o}\right)+k_{1 \min } X_{1}\left(t_{o}\right)\right\}} .
\end{aligned}
$$

This bound, Characteristic 3, and (A.16) lead to

$$
\begin{gathered}
a_{o}=k_{2} \mu_{1} X_{1} \leq k_{2 \max } \mu_{1} X_{1} \leq \bar{\kappa}_{\mu_{1} X_{1}}, \\
\bar{\kappa}_{\mu_{1} X_{1}} \\
=\frac{k_{2 \max } \mu_{1 \max }}{k_{1 \min }} \\
\times \frac{\left[\max \left\{S_{1 i \max } / \min \left\{1, \alpha_{\min }\right\}, S_{1}\left(t_{o}\right)+k_{1 \min } X_{1}\left(t_{o}\right)\right\}\right]^{2}}{K_{S 1}+\max \left\{S_{1 i \max } / \min \left\{1, \alpha_{\min }\right\}, S_{1}\left(t_{o}\right)+k_{1 \min } X_{1}\left(t_{o}\right)\right\}} .
\end{gathered}
$$

This is the constant upper bound for the acidogenic reaction rate term $k_{2} \mu_{1} X_{1}$. End of proof.

Remark A.4. The reaction rate term $k_{2} \mu_{1} X_{1}$ has a constant upper bound for any structure of the specific growth rate $\mu_{1}$, as can be concluded from the procedure shown in Proof of Lemma A.3. The reasons are (i) the concentrations $X_{1}$ and $S_{1}$ are bounded for nonnegative dilution rate $D$, as is concluded from Proof of Lemma A.1; (ii) the specific growth rates are usually bounded for bounded values of the substrate concentration, so that $\mu_{1}$ is bounded for nonnegative dilution rate $D$; and (iii) if $X_{1}$ and $\mu_{1}$ are bounded, then $k_{2} \mu_{1} X_{1}$ is also bounded.

The upper bounds for $X_{2}$ and $S_{2}$ are established as the following.

Lemma A.5. Consider the plant (1)-(5), subject to Characteristics 1 to 6 and Assumption 1. The concentrations of methanogenic biomass and VFA are bounded as follows: $X_{2} \leq$ $\bar{\kappa}_{x_{2}}, S_{2} \leq \bar{\kappa}_{S_{2}}$, where $\bar{\kappa}_{x_{2}}, \bar{\kappa}_{S_{2}}$ are positive constants defined as

$$
\begin{aligned}
& \bar{\kappa}_{x_{2}}= \frac{1}{k_{3 \min }} \\
& \times \max \left\{\frac{S_{2 i \max }}{\min \left\{1, \alpha_{\min }\right\}}+\frac{k_{2 \max } S_{1 i \max }}{k_{1 \min } \min \left\{1, \alpha_{\min }\right\}},\right. \\
&\left.S_{2}\left(t_{o}\right)+k_{3 \min } X_{2}\left(t_{o}\right)+\frac{k_{2 \max }}{k_{1 \min }} S_{1}\left(t_{o}\right)\right\},
\end{aligned}
$$

$$
\begin{aligned}
\bar{\kappa}_{S_{2}}=\max \left\{\frac{S_{2 i \max }}{\min \left\{1, \alpha_{\min }\right\}}+\frac{k_{2 \max } S_{1 i \max }}{k_{1 \min } \min \left\{1, \alpha_{\min }\right\}},\right. \\
\left.S_{2}\left(t_{o}\right)+k_{3 \min } X_{2}\left(t_{o}\right)+\frac{k_{2 \max }}{k_{1 \min }} S_{1}\left(t_{o}\right)\right\},
\end{aligned}
$$

where $S_{2 i \max }, \alpha_{\min }, k_{2 \max }, k_{1 \text { min }}$, and $k_{3 \text { min }}$ are constant bounds established in Characteristic 3. This statement is independent of the control law, the update law, and observer mechanism used.

Proof. Let

$$
Z_{2}=S_{2}+k_{3 \min } X_{2}+\left(\frac{k_{2 \max }}{k_{1 \min }}\right) S_{1}
$$

where $k_{3 \min }, k_{2 \max }$, and $k_{1 \text { min }}$ are constant bounds established in Characteristic 3. Differentiating with respect to time and using (4), (2), (3) yield

$$
\begin{aligned}
\dot{Z}_{2}= & \dot{S}_{2}+k_{3 \min } \dot{X}_{2}+\left(\frac{k_{2 \max }}{k_{1 \min }}\right) \dot{S}_{1} \\
= & S_{2}^{\text {in }} D-S_{2} D-\alpha k_{3 \min } D X_{2}+\left(\frac{k_{2 \max }}{k_{1 \text { min }}}\right) S_{1}^{\text {in }} D \\
& -\left(\frac{k_{2 \max }}{k_{1 \text { min }}}\right) S_{1} D+k_{2} \mu_{1} X_{1}-\left(\frac{k_{2 \max }}{k_{1 \text { min }}}\right) k_{1} \mu_{1} X_{1} \\
& -k_{3} \mu_{2} X_{2}+k_{3 \min } \mu_{2} X_{2} .
\end{aligned}
$$


Using the inequalities of Characteristic 3 yields

$$
\begin{aligned}
& -k_{3} \mu_{2} X_{2}+k_{3 \min } \mu_{2} X_{2} \leq 0 \text {, } \\
& \dot{Z}_{2} \leq-S_{2} D-\alpha_{\min } k_{3 \min } X_{2} D-\left(\frac{k_{2 \max }}{k_{1 \min }}\right) S_{1} D \\
& +S_{2 i \max } D \\
& +\left(\frac{k_{2 \max }}{k_{1 \min }}\right) S_{1 i \max } D+k_{2 \max } \mu_{1} X_{1} \\
& -\left(\frac{k_{2 \max }}{k_{1 \text { min }}}\right) k_{1} \mu_{1} X_{1} \\
& \leq-S_{2} D-\alpha_{\min } k_{3 \min } X_{2} D \\
& -\left(\frac{k_{2 \max }}{k_{1 \text { min }}}\right) S_{1} D+S_{2 i \text { max }} D \\
& +\left(\frac{k_{2 \max }}{k_{1 \min }}\right) S_{1 i \max } D \\
& \leq-\min \left\{1, \alpha_{\min }\right\}\left[S_{2}+k_{3 \min } X_{2}+\frac{k_{2 \max }}{k_{1 \min }} S_{1}\right. \\
& -\frac{S_{2 i \max }}{\min \left\{1, \alpha_{\min }\right\}} \\
& \left.-\frac{k_{2 \max } S_{1 i \max }}{k_{1 \min } \min \left\{1, \alpha_{\min }\right\}}\right] D \text {. }
\end{aligned}
$$

Let

$$
\begin{aligned}
\bar{Z}_{2}= & Z_{2}-\frac{S_{2 i \max }}{\min \left\{1, \alpha_{\min }\right\}}-\frac{k_{2 \max } S_{1 i \max }}{k_{1 \min } \min \left\{1, \alpha_{\min }\right\}}, \\
\bar{Z}_{2}= & S_{2}+k_{3 \min } X_{2}+\frac{k_{2 \max }}{k_{1 \min }} S_{1} \\
& -\frac{S_{2 i \max }}{\min \left\{1, \alpha_{\min }\right\}}-\frac{k_{2 \max } S_{1 i \max }}{k_{1 \min } \min \left\{1, \alpha_{\min }\right\}},
\end{aligned}
$$

where $Z_{2}$ is defined in (A.22). Expression (A.24) is rewritten in terms of $\bar{Z}_{2}$ (A.26) as the following. Differentiating (A.25) with respect to time yields $\dot{\bar{Z}}_{2}=\dot{Z}_{2}$. Using (A.24) and definition (A.26) yields $\dot{\bar{Z}}_{2} \leq-\min \left\{1, \alpha_{\min }\right\} \bar{Z}_{2} D$. Using Lemma in [28] yields $\bar{Z}_{2} \leq \bar{Z}_{2}\left(t_{o}\right) e^{-\min \left\{1, \alpha_{\min }\right\} D\left(t-t_{o}\right)}$, where $\bar{Z}_{2}\left(t_{o}\right)$ is the initial value of $\bar{Z}_{2}$ and can be obtained by evaluating (A.26) for $t=t_{o}$ :

$$
\begin{aligned}
\bar{Z}_{2}\left(t_{o}\right)= & S_{2}\left(t_{o}\right)+k_{3 \min } X_{2}\left(t_{o}\right)+\frac{k_{2 \max }}{k_{1 \min }} S_{1}\left(t_{o}\right) \\
& -\frac{S_{2 i \max }}{\min \left\{1, \alpha_{\min }\right\}}-\frac{k_{2 \max } S_{1 i \max }}{k_{1 \min } \min \left\{1, \alpha_{\min }\right\}}
\end{aligned}
$$

Thus, $\bar{Z}_{2} \leq \max \left\{0, \bar{Z}_{2}\left(t_{o}\right)\right\}$. Using the definition (A.26) yields

$$
\begin{aligned}
& S_{2}+k_{3 \min } X_{2}+\frac{k_{2 \max }}{k_{1 \min }} S_{1}-\frac{S_{2 i \max }}{\min \left\{1, \alpha_{\min }\right\}} \\
& -\frac{k_{2 \max } S_{1 i \max }}{k_{1 \min } \min \left\{1, \alpha_{\min }\right\}} \\
& \leq \max \left\{0, S_{2}\left(t_{o}\right)+k_{3 \min } X_{2}\left(t_{o}\right)+\frac{k_{2 \max }}{k_{1 \min }} S_{1}\left(t_{o}\right)\right. \\
& \left.\quad-\frac{S_{2 i \max }}{\min \left\{1, \alpha_{\min }\right\}}-\frac{k_{2 \max } S_{1 i \max }}{k_{1 \min } \min \left\{1, \alpha_{\min }\right\}}\right\}, \\
& \leq k_{3 \min } X_{2}+\frac{k_{2 \max }}{k_{1 \min }} S_{1} \\
& \leq \frac{S_{2 i \max }}{\min \left\{1, \alpha_{\min }\right\}}+\frac{k_{2 \max } S_{1 i \max }}{k_{1 \min } \min \left\{1, \alpha_{\min }\right\}}, \\
& \left.S_{2}\left(t_{o}\right)+k_{3 \min } X_{2}\left(t_{o}\right)+\frac{k_{2 \max }}{k_{1 \min }} S_{1}\left(t_{o}\right)\right\}
\end{aligned}
$$

and, hence,

$$
\begin{gathered}
X_{2} \leq \bar{\kappa}_{x_{2}} \\
\bar{\kappa}_{x_{2}}=\frac{1}{k_{3 \min }} \max \left\{\frac{S_{2 i \max }}{\min \left\{1, \alpha_{\min }\right\}}+\frac{k_{2 \max } S_{1 i \max }}{k_{1 \min } \min \left\{1, \alpha_{\min }\right\}},\right. \\
\left.S_{2}\left(t_{o}\right)+k_{3 \min } X_{2}\left(t_{o}\right)+\frac{k_{2 \max }}{k_{1 \min }} S_{1}\left(t_{o}\right)\right\} \\
\bar{\kappa}_{S_{2}}=\max \left\{\frac{S_{2 i \max } \leq \bar{\kappa}_{S_{2}}}{\min \left\{1, \alpha_{\min }\right\}}+\frac{k_{2 \max } S_{1 i \max }}{k_{1 \min } \min \left\{1, \alpha_{\min }\right\}},\right. \\
\left.S_{2}\left(t_{o}\right)+k_{3 \min } X_{2}\left(t_{o}\right)+\frac{k_{2 \max }}{k_{1 \min }} S_{1}\left(t_{o}\right)\right\}
\end{gathered}
$$

End of proof.

Remark A.6. Lemma A.5 indicates that $X_{2}, S_{2}$ are bounded if the dilution rate $D$ is non-negative, regardless the control law and observer used. In addition, the concentrations $X_{2}, S_{2}$ have a constant upper bound for any structure of the specific growth rates $\mu_{1}, \mu_{2}$, as can be concluded from the procedure shown in Proof of Lemma A.5.

The upper bound for $\mu_{2} X_{2}$ can be established on the basis of the Lemma A. 5 shown above.

Lemma A.7. Consider the plant (1)-(5), subject to Characteristics 1 to 6 and Assumption 1. The term $\mu_{2} X_{2}$ is bounded as 
follows: $\mu_{2} X_{2} \leq \bar{\kappa}_{\mu_{2} x_{2}}$, where $\bar{\kappa}_{\mu_{2} x_{2}}$ is a positive constant defined as

$$
\begin{aligned}
\bar{\kappa}_{\mu_{2} x_{2}}= & \frac{\bar{\mu}_{2 \max } \sqrt{K_{I_{2} \max }}}{k_{3 \min }\left(2 \sqrt{K_{S_{2} \min }}+\sqrt{K_{I_{2} \min }}\right)} \\
& \times \max \left\{\frac{S_{2 i \max }}{\min \left\{1, \alpha_{\min }\right\}}+\frac{k_{2 \max } S_{1 i \max }}{k_{1 \min } \min \left\{1, \alpha_{\min }\right\}},\right. \\
& \left.S_{2}\left(t_{o}\right)+k_{3 \min } X_{2}\left(t_{o}\right)+\frac{k_{2 \max }}{k_{1 \min }} S_{1}\left(t_{o}\right)\right\},
\end{aligned}
$$

where $\bar{\mu}_{2 \text { max }}, \quad K_{I_{2} \max }, K_{S_{2} \text { min }}, \quad K_{I_{2} \min }, S_{2 i \max }, \alpha_{\min }, k_{2 \max }$, $S_{1 i \max }, k_{1 \text { min }}, k_{3 \text { min }}$, and $k_{2 \max }$ are constant bounds established in Characteristic 3. This statement is independent of the control law, the update law, and observer mechanism used.

Proof. The maximum value of $\mu_{2}$ can be found by differentiating it with respect to $S_{2}$. Differentiating $\mu_{2}$ (5) with respect to $S_{2}$ yields

$$
\frac{\partial \mu_{2}}{\partial S_{2}}=\mu_{o} \frac{K_{S_{2}}-S_{2}^{2} / K_{I_{2}}}{\left(K_{S_{2}}+S_{2}+S_{2}^{2} / K_{I_{2}}\right)^{2}}
$$

Equating to zero, solving for $S_{2}$, and substituting in (5) yield

$$
\begin{aligned}
& S_{2}^{*}=\sqrt{K_{I_{2}} K_{S_{2}}}, \\
& \mu_{2}^{*}=\mu_{2 \max } \frac{\sqrt{K_{I_{2}}}}{2 \sqrt{K_{S_{2}}}+\sqrt{K_{I_{2}}}} .
\end{aligned}
$$

The optimal value $S_{2}^{*}=\sqrt{K_{I_{2}} K_{S_{2}}}$ was already established in [41]. Since $\mu_{2 \max }, K_{I_{2}}, K_{S_{2}}$ may be time-varying, $\mu_{2}$ (A.34) has an upper bound:

$$
\begin{aligned}
\mu_{2} & \leq \mu_{2 \max } \frac{\sqrt{K_{I_{2}}}}{2 \sqrt{K_{S_{2}}}+\sqrt{K_{I_{2}}}} \\
& \leq \bar{\mu}_{2 \max } \frac{\sqrt{K_{I_{2} \max }}}{2 \sqrt{K_{S_{2} \min }}+\sqrt{K_{I_{2} \min }}}
\end{aligned}
$$

where $\bar{\mu}_{2 \text { max }}, K_{I_{2} \max }, K_{I_{2} \text { min }}$, and $K_{S_{2} \text { min }}$ are established in Characteristic 3. Therefore, using (A.29) and property (A.35) yields

$$
\begin{gathered}
\mu_{2} X_{2} \leq \bar{\kappa}_{\mu_{2} x_{2}}, \\
\bar{\kappa}_{\mu_{2} x_{2}}=\frac{\bar{\mu}_{2 \max } \sqrt{K_{I_{2} \max }},}{k_{3 \min }\left(2 \sqrt{K_{S_{2} \min }}+\sqrt{K_{I_{2} \min }}\right)} \\
\times \max \left\{\frac{S_{2 i \max }}{\min \left\{1, \alpha_{\min }\right\}}+\frac{k_{2 \max } S_{1 i \max }}{k_{1 \min } \min \left\{1, \alpha_{\min }\right\}},\right. \\
\left.S_{2}\left(t_{o}\right)+k_{3 \min } X_{2}\left(t_{o}\right)+\frac{k_{2 \max }}{k_{1 \min }} S_{1}\left(t_{o}\right)\right\}
\end{gathered}
$$

End of proof.

Remark A.8. The reaction rate term $\mu_{2} X_{2}$ has a constant upper bound for any structure of the specific growth rates $\mu_{1}$ and $\mu_{2}$, as is concluded from the procedure in the above proof. The reasons are (i) the biomass concentration $S_{2}$ is bounded for nonnegative dilution rate $D$, as is indicated by Lemma A.5, (ii) the specific growth rates are usually bounded for bounded values of the substrate concentration, so that $\mu_{2}$ is bounded for nonnegative dilution rate $D$, and (iii) the biomass concentration $X_{2}$ is bounded for non-negative dilution rate $D$, as is indicated by Lemma A.5.

Remark A.9. Lemmas A.1, A.3, A.5, and A.7 establish the results stated in Lemma A.3.1.

\section{B. Proof of Property (20)}

Solving (18) for $z$ yields

$$
\begin{gathered}
z= \pm\left(C_{b z}+\sqrt{2 V_{z}}\right) \quad \text { if } V_{z} \geq 0, \\
z \in\left[-C_{b z}, C_{b z}\right] \quad \text { if } V_{z}=0 .
\end{gathered}
$$

Therefore,

$$
\begin{gathered}
|z|=\left|C_{b z}+\sqrt{2 V_{z}}\right| \quad \text { if } V_{z} \geq 0 \\
|z| \in\left[0, C_{b z}\right], \quad \text { if } V_{z}=0 .
\end{gathered}
$$

Hence,

$$
|z| \leq C_{b z}+\sqrt{2 V_{z}}
$$

End of proof.

\section{Proof of Property (41)}

The signal $\left|g_{z}\right|$ can be rewritten as

$$
\left|g_{z}\right|=g_{z} \operatorname{sgn}\left(g_{z}\right) .
$$


From (24), it follows that

$$
\begin{gathered}
\operatorname{sgn}\left(g_{z}\right)=\operatorname{sgn}(z) \quad \text { if } z>C_{b z} \text { or } z<-C_{b z}, \\
g_{z}=0 \quad \text { if } z \in\left[-C_{b z}, C_{b z}\right], \\
\left|g_{z}\right|=0 \quad \text { if } z \in\left[-C_{b z}, C_{b z}\right] .
\end{gathered}
$$

From (42) it follows that

$$
\operatorname{sat}_{z}=\operatorname{sgn}(z) \text { if }|z|>C_{b z} .
$$

From expressions (C.1), (C.2), (C.4), and (C.5) it follows that

$$
\begin{gathered}
\operatorname{sgn}\left(g_{z}\right)=\operatorname{sgn}(z)=\operatorname{sat}_{z} \quad \text { if }|z|>C_{b z}, \\
\left|g_{z}\right|=g_{z} \operatorname{sgn}(z)=g_{z} \text { sat }_{z} \quad \text { if }|z|>C_{b z}, \\
\left|g_{z}\right|=0=g_{z} \text { sat }_{z} \quad \text { if } z \in\left[-C_{b z}, C_{b z}\right] .
\end{gathered}
$$

Combining the last two expressions yields $\left|g_{z}\right|=g_{z}$ sat $_{z}$. End of proof.

\section{Conflict of Interests}

The authors declare that there is no conflict of interests regarding the publication of this paper.

\section{Acknowledgment}

This work was partially supported by Universidad Nacional de Colombia-Manizales, Project 16074, Vicerrectoría de Investigación, DIMA.

\section{References}

[1] H. Wang, B. Chen, X. Liu, K. Liu, and C. Lin, "Robust adaptive fuzzy tracking control for pure-feedback stochastic nonlinear systems with input constraints," IEEE Transactions on Cybernetics, vol. 43, no. 6, pp. 2093-2104, 2013.

[2] H. Wang, B. Chen, and C. Lin, "Approximation-based adaptive fuzzy control for a class of non-strict-feedback stochastic nonlinear systems," Science China Information Sciences, vol. 57, no. 3, pp. 1-16, 2014.

[3] S. Tong, Y. Li, Y. Li, and Y. Liu, "Observer-based adaptive fuzzy backstepping control for a class of stochastic nonlinear strictfeedback systems," IEEE Transactions on Systems, Man, and Cybernetics B: Cybernetics, vol. 41, no. 6, pp. 1693-1704, 2011.

[4] S.-C. Tong, X.-L. He, and H.-G. Zhang, "A combined backstepping and small-gain approach to robust adaptive fuzzy output feedback control," IEEE Transactions on Fuzzy Systems, vol. 17, no. 5, pp. 1059-1069, 2009.

[5] S. Tong and Y. Li, "Adaptive fuzzy output feedback tracking backstepping control of strict-feedback nonlinear systems with unknown dead zones," IEEE Transactions on Fuzzy Systems, vol. 20, no. 1, pp. 168-180, 2012.

[6] R. Aguilar-López and R. Maya-Yescas, "State estimation for nonlinear systems under model uncertainties: a class of slidingmode observers," Journal of Process Control, vol. 15, no. 3, pp. 363-370, 2005.

[7] J. H. Ahrens and H. K. Khalil, "High-gain observers in the presence of measurement noise: a switched-gain approach," Automatica, vol. 45, no. 4, pp. 936-943, 2009.
[8] L. K. Vasiljevic and H. K. Khalil, "Error bounds in differentiation of noisy signals by high-gain observers," Systems and Control Letters, vol. 57, no. 10, pp. 856-862, 2008.

[9] A. Levant, "Robust exact differentiation via sliding mode technique," Automatica, vol. 34, no. 3, pp. 379-384, 1998.

[10] G. Goffaux, A. Vande Wouwer, and O. Bernard, "Improving continuous-discrete interval observers with application to microalgae-based bioprocesses," Journal of Process Control, vol. 19, no. 7, pp. 1182-1190, 2009.

[11] M. Moisan, O. Bernard, and J.-L. Gouzé, "Near optimal interval observers bundle for uncertain bioreactors," Automatica, vol. 45, no. 1, pp. 291-295, 2009.

[12] V. Alcaraz-González, J. Harmand, A. Rapaport, J. P. Steyer, V. González-Alvarez, and C. Pelayo-Ortiz, "Software sensors for highly uncertain WWTPs: a new approach based on interval observers," Water Research, vol. 36, no. 10, pp. 2515-2524, 2002.

[13] I. Kanellakopoulos, "Robustification tools for nonlinear control design," in Proceedings of the 33rd IEEE Conference on Decision and Control, pp. 3464-3468, Lake Buena Vista, December 1994.

[14] O. Bernard, Z. Hadj-Sadok, D. Dochain, A. Genovesi, and J.-P. Steyer, "Dynamical model development and parameter identification for an anaerobic wastewater treatment process," Biotechnology and Bioengineering, vol. 75, no. 4, pp. 424-438, 2001.

[15] H. Méndez-Acosta, B. Palacios-Ruiz, V. Alcaraz-González, J. Steyer, V. González-Álvarez, and E. Latrille, "Robust control of volatile fatty acids in anaerobic digestion processes," Industrial \& Engineering Chemistry Research, vol. 47, no. 20, pp. 77157720, 2008.

[16] H. Méndez-Acosta, B. Palacios-Ruiz, V. Alcaraz-González, V. González-Álvarez, and J. García-Sandoval, "A robust control scheme to improve the stability of anaerobic digestion processes," Journal of Process Control, vol. 20, no. 4, pp. 375-383, 2010.

[17] M. Estaben, M. Polit, and J. P. Steyer, "Fuzzy control for an anaerobic digester," Control Engineering Practice, vol. 5, no. 9, pp. 1303-1310, 1997.

[18] L. Mailleret, O. Bernard, and J. P. Steyer, "Robust regulation of anaerobic digestion processes," Water Science and Technology, vol. 48 , no. 6, pp. 87-94, 2003.

[19] J. Hess and O. Bernard, "Design and study of a risk management criterion for an unstable anaerobic wastewater treatment process," Journal of Process Control, vol. 18, no. 1, pp. 71-79, 2008.

[20] N. Hilgert, J. Harmand, J.-P. Steyer, and J.-P. Vila, "Nonparametric identification and adaptive control of an anaerobic fluidized bed digester," Control Engineering Practice, vol. 8, no. 4, pp. 367376, 2000.

[21] L. Mailleret, O. Bernard, and J.-P. Steyer, "Nonlinear adaptive control for bioreactors with unknown kinetics," Automatica, vol. 40, no. 8, pp. 1379-1385, 2004.

[22] H. O. Méndez-Acosta, D. U. Campos-Delgado, R. Femat, and V. González-Alvarez, "A robust feedforward/feedback control for an anaerobic digester," Computers and Chemical Engineering, vol. 29, no. 7, pp. 1613-1623, 2005.

[23] B. Chachuat and O. Bernard, "Probabilistic observers for a class of uncertain biological processes," International Journal of Robust and Nonlinear Control, vol. 16, no. 3, pp. 157-171, 2006.

[24] R. Aguilar-López, R. Acevedo-Gómez, M. Neria, and A. Domínguez-Bocanegra, "State variables monitoring using a class of nonlinear observer based estimator, applied to continuous biosystem," Journal of Applied Research and Technology, vol. 6, no. 3, pp. 147-158, 2008. 
[25] E. Aguilar-Garnica, D. Dochain, V. Alcaraz-González, and V. González-Álvarez, "A multivariable control scheme in a twostage anaerobic digestion system described by partial differential equations," Journal of Process Control, vol. 19, no. 8, pp. 13241332, 2009.

[26] M. I. Neria-González, R. Martínez-Guerra, and R. AguilarLópez, "Feedback regulation of an industrial aerobic wastewater plant," Chemical Engineering Journal, vol. 139, no. 3, pp. 475-481, 2008.

[27] M. M. Polycarpou and P. A. Ioannou, "On the existence and uniqueness of solutions in adaptive control systems," IEEE Transactions on Automatic Control, vol. 38, no. 3, pp. 474-479, 1993.

[28] J. Slotine and W. Li, Applied Nonlinear Control, Prentice Hall, Englewood Cliffs, NJ, USA, 1991.

[29] A. Leva, L. Piroddi, M. Di Felice, A. Boer, and R. Paganini, "Adaptive relay-based control of household freezers with on-off actuators," Control Engineering Practice, vol. 18, no. 1, pp. 94-102, 2010.

[30] M. D. Felice, L. Piroddi, A. Leva, and A. Boer, "Adaptive temperature control of a household refrigerator," in Proceedings of the American Control Conference (ACC '09), pp. 889-894, St. Louis, Mo, USA, June 2009.

[31] J.-W. Choi and S.-C. Lee, "Antiwindup strategy for PI-type speed controller," IEEE Transactions on Industrial Electronics, vol. 56, no. 6, pp. 2039-2046, 2009.

[32] A. Rincon, C. Erazo, and F. Angulo, "A robust adaptive controller for an anaerobic digester with saturated input: guarantees for the boundedness and convergence properties," Journal of Process Control, vol. 22, no. 9, pp. 1785-1792, 2012.

[33] K.-M. Koo, "Stable adaptive fuzzy controller with time-varying dead-zone," Fuzzy Sets and Systems, vol. 121, no. 1, pp. 161-168, 2001.

[34] X.-S. Wang, C.-Y. Su, and H. Hong, "Robust adaptive control of a class of nonlinear systems with unknown dead-zone," Automatica, vol. 40, no. 3, pp. 407-413, 2004.

[35] Y. Tan and I. Kanellakopoulos, "Adaptive nonlinear friction compensation with parametric uncertainties," in Proceedings of the American Control Conference (ACC '99), pp. 2511-2515, June 1999.

[36] P. Ioannou and J. Sun, Robust Adaptive Control, Prentice-Hall PTR Upper, Upper Saddle River, NJ, USA, 1996.

[37] A. Rincon, F. Angulo, and G. Olivar, "Control of an anaerobic digester through normal form of fold bifurcation," Journal of Process Control, vol. 19, no. 8, pp. 1355-1367, 2009.

[38] M. Sbarciog, M. Loccufier, and E. Noldus, "Determination of appropriate operating strategies for anaerobic digestion systems," Biochemical Engineering Journal, vol. 51, no. 3, pp. 180188, 2010.

[39] C. García-Diéguez, F. Molina, and E. Roca, "Multi-objective cascade controller for an anaerobic digester," Process Biochemistry, vol. 46, no. 4, pp. 900-909, 2011.

[40] H. Méndez-Acosta, R. Femat, V. González-Alvarez, and J. Steyer, "Substrate regulation in an anaerobic digester based on geometric control," in Proceedings of the CLAC-IFAC, Mexico, México, 2002.

[41] P. Cougnon, D. Dochain, M. Guay, and M. Perrier, "On-line optimization of fedbatch bioreactors by adaptive extremum seeking control," Journal of Process Control, vol. 21, no. 10, pp. 1526-1532, 2011. 


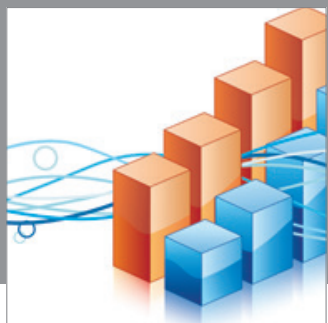

Advances in

Operations Research

mansans

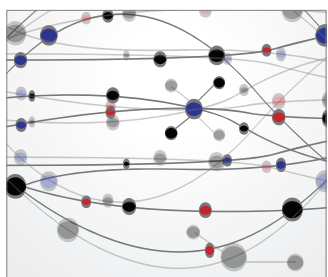

The Scientific World Journal
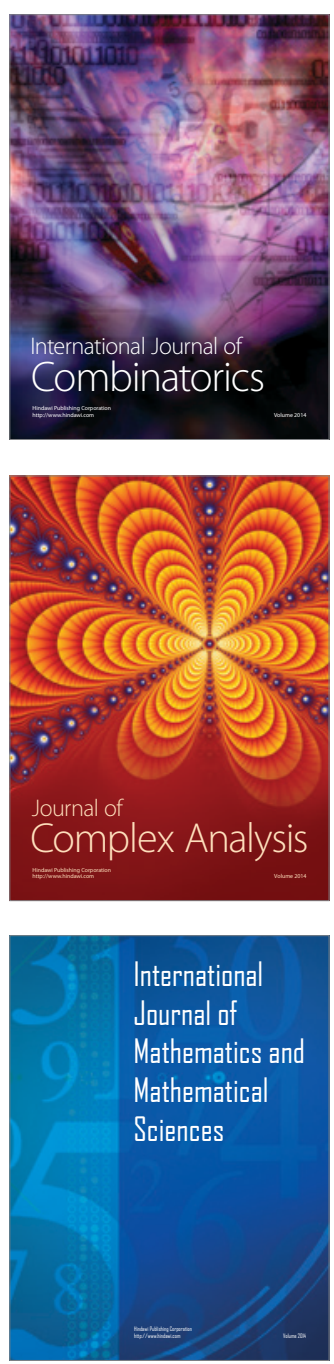
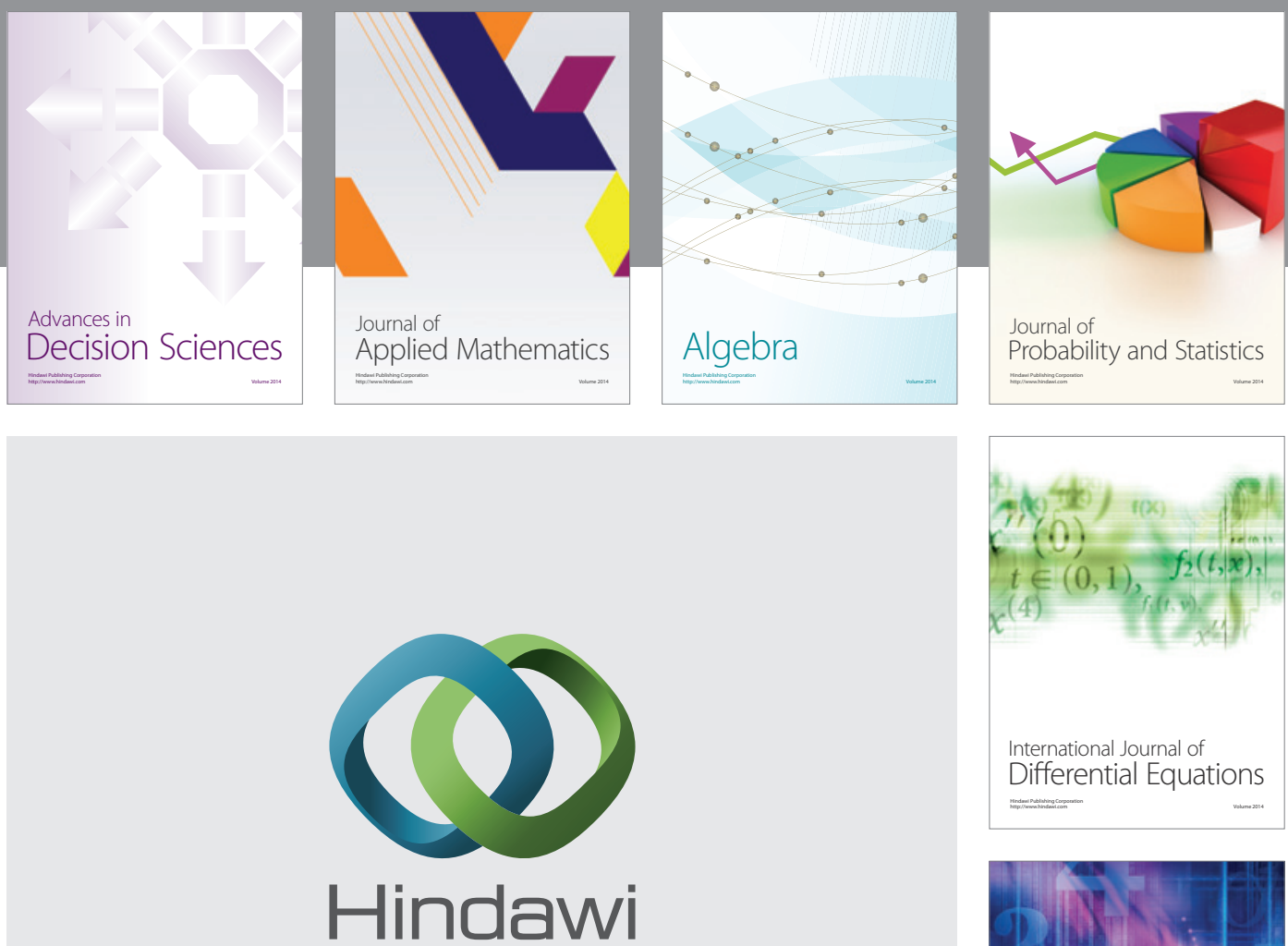

Submit your manuscripts at http://www.hindawi.com
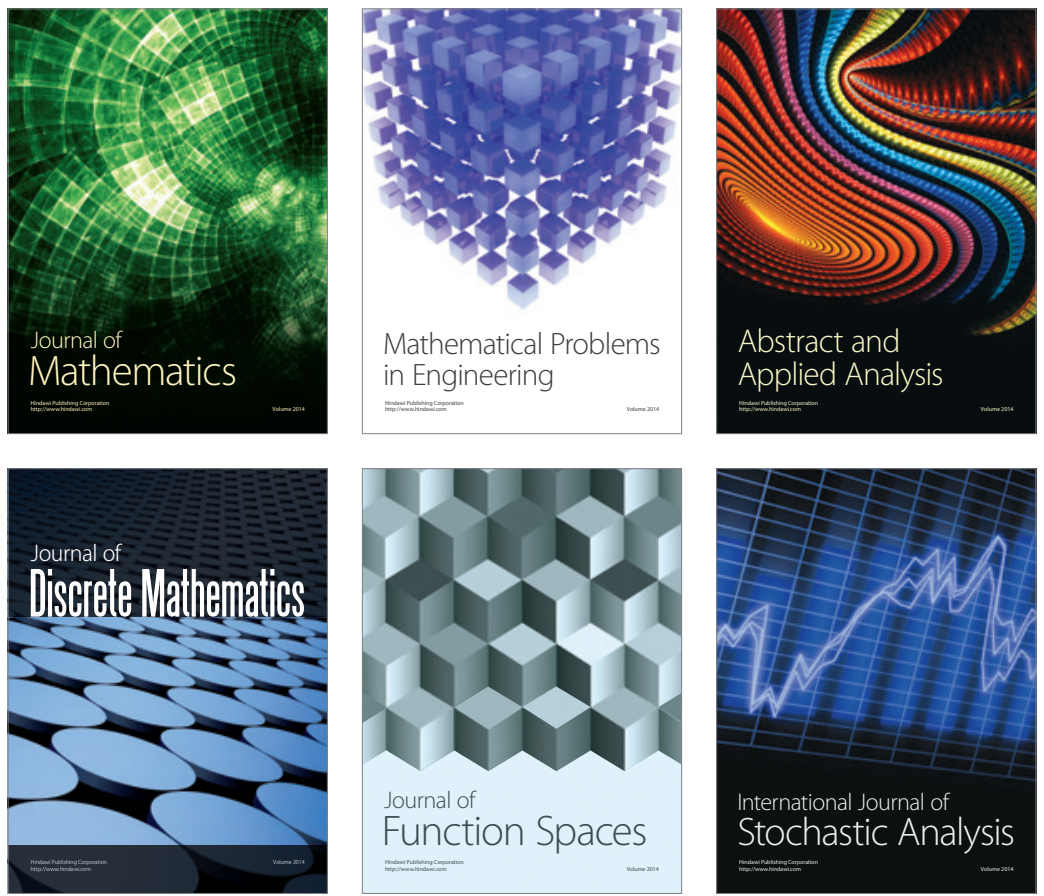

Journal of

Function Spaces



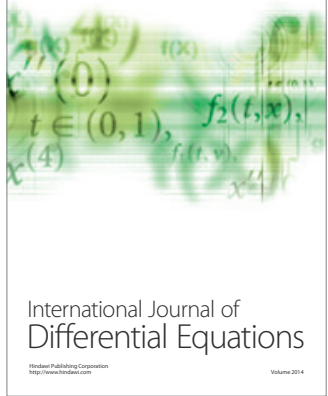
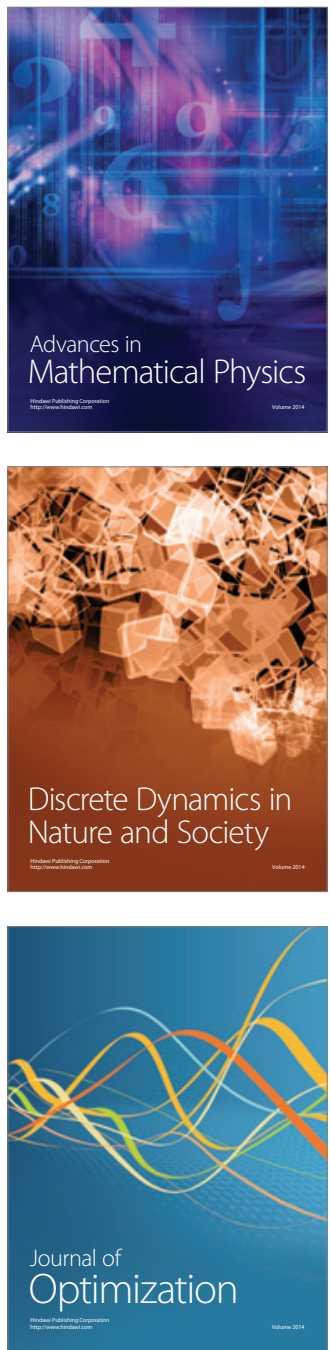